\title{
Rechtliche Aspekte von Generationengerechtigkeit
}

In derpolitischen Diskussion und Gesetzgebung erscheint in den letzten Jabren immer häufiger der Begriff der Generationengerechtigkeit. ${ }^{1}$ Es ist ungeklärt, was darunter zu verstehen ist. Hier sollen aus rechtlicher Sicht einige Aspekte aufgezeigt werden.

\section{Generationengerechtigkeit als Rechtsbegriff?}

Was kann das Recht zur Debatte um Generationengerechtigkeit beitragen? Bei Gerechtigkeit scheint die Kernkompetenz der Rechtswissenschaft angesprochen. Gerechtigkeit ist zusammen mit Zweckmäßigkeit und Rechtssicherheit zentrale Aufgabe des Rechts. ${ }^{2}$

Schwieriger ist es, den Begriff der Generationen im Recht einzuordnen. Im Text des Grundgesetzes erscheint er in dem 1994 eingefügten Art. 20 a:

"Der Staat schützt auch in Verantwortung für die künftigen Generationen die natürlichen Lebensgrundlagen und die Tiere im Rabmen der verfassungsmäßigen Ordnung durch die Gesetzgebung und nach Maßgabe von Gesetz und Recht durch die vollziehende Gewalt und die Rechtsprechung."

Damit sind Politik und Recht der natürlichen Umwelt angesprochen, eines der politischen Themen, die mit dem Generationenbegriff verbunden werden. Die anderen sind die soziale Sicherung im Alter und für Familien sowie die Haushaltspolitik. ${ }^{3}$ Für jeden dieser drei Bereiche wird der Begriff »Generation « unterschiedlich verstanden und in verschiedene politische und normative Kontexte gestellt. Gemeinsam ist ihnen, dass sie längerfristig wirksame Entscheidungen betreffen.

Der Generationenbegriff ist aber auch schillernd und ungenau und kann darum wie zu zeigen ist - das Verständnis von Problemen auch erschweren. Das Recht ist insgesamt nur schlecht auf die Gerechtigkeit zwischen Kollektivsubjekten eingerichtet. So gehören die Gerechtigkeit zwischen den Geschlechtern, Völkern oder Religionsgemeinschaften zu den schwierigsten und konfliktträchtigsten Gleichheiten. Soziale Gerechtigkeit zwischen ökonomischen Klassen ist nach wie vor eine ungelöste, vielleicht unlösbare Aufgabe des Sozialstaatsprinzips. ${ }^{4}$ Schritte zur kollektiven Gerechtigkeit bringen stets die Gefahr, individuelle Gerechtigkeit zu verlieren. Ein Kollektivsubjekt braucht hinreichend homogene Interessen und Lebenslagen, damit zumindest dessen große Mehrheit sich durch die kollektive Gerechtigkeit auch vertreten fühlt. Die Generation als kollektives Subjekt ist je-

1 Vgl. BT-Drucks. 15/2149 vom 9. Dezember 2003, Fraktionen SPD und Bündnis 90/Die Grünen, Entwurf eines Gesetzes zur Sicherung der nachhaltigen Finanzierungsgrundlagen der gesetzlichen Rentenversicherung, S. 1, 17: »Richtschnur für diese Reformmaßnahmen ist dabei der Grundsatz der Generationengerechtigkeit. « Bereits BT-Drucks. 14/4595, S. 85; Vgl. weiter Tremmel, ZRP 2004, S. 44 ff.; Däubler-Gmelin, ZRP 2000, S. 27 ff.; Butterwegge/Klundt; Von Lucke, Blätter für deutsche und internationale Politik 2003, S. $1055 \mathrm{ff}$.

2 Radbruch, S. 54, 73 ff.; Heller, S. 216 ff.

3 Art. 115 Abs. 1 Satz 2 GG.

4 Vgl. Heller, S. $166 \mathrm{ff}$. 
doch nicht eindeutig definiert: ${ }^{5} \mathrm{Nimmt}$ man die jeweils alte oder junge Generation in den Blick, so lassen sich gemeinsame Lebenslagen identifizieren, aber sie besteht aus stets wechselnden Individuen. Nimmt man einen Geburtsjahrgang als Generation, so bleiben ihre Mitglieder identisch, ihre Ausgangspositionen sind aber bereits verschieden, ${ }^{6}$ und ihre Interessen und Lebenslagen wechseln. So ist die Generation wohl nur für wenige Felder der Gerechtigkeit ein taugliches Kollektivsubjekt.

\section{Rechtliche Vorgaben für den Ausgleich zwischen den Generationen}

\section{Umweltstaat}

Ausgangspunkt ist das schon zitierte Umweltstaatsgebot. Hier ist nicht völlig klar, wer die künftigen Generationen sind. Zunächst nützt der Schutz natürlicher Lebensgrundlagen auch uns als heutiger Generation. Art. 20 a GG bewirkt aber, dass auch die heute noch nicht geborenen Menschen als Adressaten heutiger Politik betrachtet werden müssen. Auch für unsere noch nicht geborenen Kinder und Enkel sowie alle nachfolgenden Generationen sollen die natürliche Artenvielfalt bewahrt, Klima und Erdatmosphäre geschützt, Wasser, Luft und Boden von Umweltgiften freigehalten werden und auch auf lange Frist wenig Schäden und Gefahren etwa in Form von Atommüll oder langlebigen Chemikalien hinterlassen werden. ${ }^{7}$ Mit dem so formulierten Umweltstaatsgebot wird ausgedrückt, dass es für den Schutz der natürlichen Lebensgrundlagen unzureichend sein kann, sich allein am kurzfristigen Wohlstandsgewinn, an bereits lebenden Rechtssubjekten und an der demokratischen Willensbildung des heutigen Volkes zu orientieren. Bei den künftigen Generationen handelt es sich insofern schlicht um die künftige Menschheit und ihr Gesamtinteresse. ${ }^{8}$

Gemessen an diesem existenziellen Anspruch scheint der normative Ertrag von Art. 20 a GG gering. Jedenfalls bislang werden Gesetzgebung, vollziehende Gewalt und Rechtsprechung nicht hinreichend wirksam kontrolliert, ob sie das neue Staatsziel beachten. Es wird oft nur als ein Belang unter vielen gewichtet. ${ }^{9}$ Konkrete Handlungsaufträge werden ihm bislang nicht entnommen..$^{10}$ Aber die Interessen künftiger Menschen können eben nur so gut beachtet werden, wie es die heutigen Menschen in den Institutionen durchsetzen. ${ }^{11} \mathrm{Ob}$ dies durch eine Neuformulierung auf »nachrückende Generationen « und ein daraus angeblich folgendes Klagerecht von Kindern und Jugendlichen geändert werden kann, wie Jörg Tremmel meint, ${ }^{12}$ kann bezweifelt werden. ${ }^{13}$ Ein Klagerecht gesetzlicher Vertreter

5 Vgl. zu den Bedeutungspotenzialen: Häberle, FS Zacher, S. 215, 225 ff.; Nullmeier in: VDR, S. 65, 67; Nullmeier, KJ 1997, S. 261, 262.

6 In der »Generation Golf« war es nur eine kleine Minderheit, die das entsprechende Fahrzeug zum 18. Geburtstag als Cabrio von den Eltern geschenkt bekommen hat.

7 Murswiek, NVwZ 1996, S. 222, 225; Kloepfer, DVBl. 1996, S. 73, 78; Epiney, in: von Mangoldt/Klein/ Starck, Das Bonner Grundgesetz, Rz. 31 zu Art. 20 a.

8 Vgl. Häberle, FS Zacher, S. 215, 217 ff.

9 So vom BVerwG für die Bauleitplanung, BVerwG vom 15. Oktober 2002, NVwZ-RR 2003, S. 171; Murswiek, NVwZ 1996, S. 222, 229.

10 Zur Begründung von Handlungsaufträgen aus Art. 20 a GG: Behrends, KJ 2000, S. 376 ff.

11 So auch Däubler-Gmelin, ZRP 2000, S. 27, 28.

12 ZRP 2004, S. 44, 46.

13 Dass die heute lebenden Generationen Adressaten sind, dürrte unstrittig sein, vgl. Peters, NVwZ 1995, 555. 
für ihre Kinder existiert schon heute, ${ }^{14}$ die Frage der subjektiven Betroffenheit durch Staatsziele würde nicht berührt. ${ }^{15}$

Entscheidend ist zunächst, ob die bestehenden Rechte wahrgenommen werden und zu materiellen Erfolgen führen. Immerhin gibt es im Umweltrecht das Vorsorgeprinzip, um bei Planungsentscheidungen und der Kontrolle der Wirtschaft auch die Interessen künftiger Generationen besser zu beachten. ${ }^{16}$ Die langfristigen ökologischen Interessen sind so in Rechtsetzung und Rechtsanwendung ein legitimer Grund, um notwendige Beschränkungen von Freiheit und Eigentum heute lebender Umweltzerstörer durchzusetzen, und werden auch argumentativ so eingesetzt. ${ }^{17}$ Wenn eine Regierung oder Gesetzgebungsmehrheit dieses Ziel ernsthaft verfolgte, wäre Art. 20 a GG hilfreich für die von ihr auszutragenden rechtlichen Konflikte, auch ohne eine umfangreiche Ergänzung der Norm. ${ }^{18}$

Es bleibt festzuhalten, dass der Schutz der natürlichen Lebensgrundlagen der eindeutigste Fall anzuwendender Generationengerechtigkeit ist. Damit ist er auch ein Test auf die Glaubwürdigkeit jeder politischen Kraft, die Generationengerechtigkeit in Anspruch nimmt. Wer bei der Rentenpolitik vom Jahr 2030 spricht, ${ }^{19}$ aber beim Klimaschutz vor wirksamen Festlegungen bis zum Jahr 2012 und darüber hinaus zurückschreckt, kann sich weder auf die Wertungen des Grundgesetzes noch auf die wirkliche Problemlage der Welt berufen.

\section{Sozialstaat}

Das Sozialstaatsgebot ist zwar nur durch das Adjektiv »sozial « in Art. 20 GG verankert, gehört aber zu den unabänderbaren und historisch fundierten grundlegenden Staatszielen, die zugleich inzwischen Europäische Gemeinschaftsziele sind..$^{20}$ Es steht für die aktiv gestaltende Rolle des Staates in der Gesellschaft und dafür, dass Staat und Recht Lebenslagen und Risiken aller Bürgerinnen und Bürger nicht ignorieren dürfen, ${ }^{21}$ sondern im Sinne sozialer Gerechtigkeit zu gestalten haben. ${ }^{22}$

In der Gesellschaft findet der soziale Staat die Tatsache vor, dass die Menschen in Generationen leben. In Kindheit und Jugend sowie im Alter sind sie nicht in der Lage, ihren Lebensbedarf selbst zu decken, sondern sind auf Unterstützung angewiesen. Die Familie als vorgefundene soziale Einheit ist hierzu nur unvollkommen in der Lage. Mit der Industrialisierung wurden hauswirtschaftliche familiäre Einheiten in Landwirtschaft und Handwerk von der Regel zur Ausnahme. Der

Vgl. die Verfahren über unzureichende Maßnahmen gegen Ozonbelastung, BVerfG vom 29. November 1995, NJW 1996, S. 651; VG Frankfurt am Main vom 21. Mai 1996, NVwZ-RR 1997, S. 92, 95, dazu Beaucamp, JA 1999, S. $170 \mathrm{ff}$.

15 Murswiek, NVwZ 1996, S. 222, 230; Steinberg, NJW 1996, S. 1985, 1992; zu einem neu zu schaffenden Umweltgrundrecht vgl. Kotulla, KJ 2000, S. $22 \mathrm{ff}$.

$16 \int 5$ Abs. 1 Nr. 2 BImSchG, $\ 7$ Abs. 2 Nr. 3 AtG; Murswiek, NVwZ 1996, S. 222, 229.

17 Vgl. BVerfG vom 10. Oktober 1997, NJW 1998, S. 367, 368 (Landschaftsschutz statt Kiesabbau); OVG Lüneburg vom 21. August 2002, ZNER 2002, Nr. 4, S. 313 (Anschluss- und Benutzungszwang für Fernwärme); VGH Mannheim vom 3. September 2002, NVwZ-RR 2003, S. 103 (Untersuchungsanordnung im Bodenschutz); OVG Brandenburg vom 31. Juli 2003, AbfallR 2003, S. 305 (Anschluss- und Benutzungszwang zu Kläranlagen); OVG Berlin vom 21. Mai 2003, Az. 1 B 1.02 (Bevorzugung von Car-Sharing bei Bewohnerparkausweisen); Peters, NVwZ 1995, S. 555, 556; Sellmann, NVwZ 2003, S. $1417,1419$.

18 Gefordert von Tremmel in: SzRG, S. 349, $364 \mathrm{f}$.

19 BT-Drucks. 15/2149 vom 9. Dezember 2003, S. $32 \mathrm{ff}$.

20 Art. 2 EGV; vgl. ausführlich Kingreen, S. $378 \mathrm{ff}$.

21 Broß, JZ 2003, S. 874, 875 zitiert Brun-Otto Bryde: »Wenn wir die soziale Wirklichkeit nicht wahrnehmen, können wir sie rechtlich nicht bewältigen - und dann haben wir auch nicht recht.«.

22 Vgl. BVerfG vom 22. Juni 1977, BVeríGE 45, 376, 387; BVerfG vom 13. Januar 1976, BVerfGE 41, 126, 153; Schulin/Igl, Rz. 21. 
Mensch als freie Arbeitskraft soll qualifiziert und mobil sein, zugleich bleibt ihm das Risiko, erwerbslos zu werden. Der Staat des 19. Jahrhunderts musste darauf mit Institutionen reagieren, die für die junge und die alte Generation sorgten. ${ }^{23}$ Dies waren zunächst die Armenfürsorge, dann die obligatorische Schule für die junge ${ }^{24}$ und schließlich die Rentenversicherung für die alte Generation. Im 20. Jahrhundert kamen für die junge Generation die Jugendhilfe und ein umfangreiches Ausbildungssystem dazu.

Der Sozialstaat Bundesrepublik hat den generationellen Ausgleich zu einem differenzierten System entwickelt, in dem mit Kindergeld und Steuerfreibeträgen sowie Ausbildungsförderung für die junge Generation und mit der dynamischen Rente, Grundsicherung im Alter und Pflegeversicherung sowohl eine Mindestsicherung wie auch am Lebensstandard orientierte Elemente enthalten sind. Dieser sozialstaatliche Generationenausgleich orientiert sich an der jeweils aktuell durch eine Lebenslage verbundenen Generation, nicht an der Kohorte des Geburtsjahrgangs. Er hat sich seit dem 19. Jahrhundert dynamisch mit der Gesellschaft und ihren politischen Kräften und Anschauungen gewandelt. So hat kein Geburtsjahrgang erlebt, dass die sozialstaatlichen Institutionen, mit denen er aufgewachsen ist, unverändert geblieben sind. ${ }^{25}$

Vielmehr hat sich der Generationenausgleich in den letzten 200 Jahren verdichtet und weiterentwickelt. Die verstärkte staatliche Intervention folgte den gesellschaftlichen Entwicklungen: ${ }^{26}$ Die Produktivkräfte erfordern eine immer besser ausgebildete junge Generation. Dass Arbeitsleben und Arbeitsmarkt mehr und mehr die Frauen einbeziehen und allen Flexibilität abverlangen, vermindert die unbezahlte Fürsorge der Familie für junge und alte Menschen und führt zu mehr gewollter und ungewollter Kinderlosigkeit. Die gewachsene Lebenserwartung und der medizinische Fortschritt verallgemeinern und verlängern - zumindest zunächst - altersbedingte Einkommenslosigkeit und Pflegebedürftigkeit. Der Sozialstaat hat Jugend und Alter zu relativen Größen gemacht.

Alle diese gesellschaftlichen Entwicklungen können ebenso als Wohltat wie als Plage wirken: So ist das wachsende Niveau benötigter Bildung und Qualifikation ebenso ein gesellschaftlicher Reichtum, wie es eine bis zum Äußersten ausgedehnte und unsichere Jugend- und Bildungsphase hervorbringt und Verlierer eines ausleseorientierten Bildungssystems produziert. Dass sich Familien verändern und vielfältiger werden, befreit von hergebrachten $Z$ wängen, führt aber auch zu materieller und emotionaler Not, wenn alte Sicherungen nicht mehr wirken, aber neue nicht in Sicht sind. In einem auf oft mehr als 20 Jahre ausgedehnten Ruhestand können sich die Menschen in ungekannter Weise entfalten, aber auch unfreiwillig aus dem Arbeitsleben und aus anderen gesellschaftlichen Bezügen ausgegrenzt werden. ${ }^{27}$ Der Sozialstaat hat die Gesellschaft integrieren können, weil er diese im Kern ökonomisch induzierten gesellschaftlichen Entwicklungen weder gestoppt noch ignoriert hat, sondern den gesellschaftlichen Widersprüchen eine Bewegungsform gegeben hat, so dass ihre Synthese als sozialer Fortschritt empfunden werden konnte. In diesem Sinne wird das Sozialstaatsprinzip als soziales Rückschrittsverbot interpretiert. ${ }^{28}$ Damit gewinnt es zugleich Bedeutung für die Ge-

25 Zur Nichtvergleichbarkeit von Gesetzeslagen, die verschiedene Jahrgänge treffen, vgl. BVerfG vom 16. Dezember 1997, BVerfGE 97, 103, 114 (Kindererziehungszeiten für Jahrgänge vor und nach 1921); BVerfG vom 14. Oktober 1997, BVerfGE 96, 330, 341 (Änderung des BAFöG 1990); SG Karlsruhe vom 18. März 2003, Az. S 6 RA 2844/02 (Kindererziehungszeiten vor und nach 1992); LSG NordrheinWestfalen, NZS 2002, S. 265, 267.

26 Für die Bundesrepublik beschrieben bei Zacher, S. $498 \mathrm{ff}$.

27 Vgl. zur Umgestaltung des Alters durch Soziaipolitik: Göckenjan, S. $298 \mathrm{ff}$.

28 Im Einzelnen sehr differenziert: Schlenker. 
neration im Sinne der Kohorte: Veränderungen akzeptiert und gestaltet, wer erwarten kann, dass sie die eigene Zukunft und diejenige der Kinder und Enkel verbessern werden. Darum reagiert der Sozialstaat nicht nur auf ökonomische und gesellschaftliche Dynamik, sondern er schafft zugleich ihre Voraussetzungen. ${ }^{29}$ Insofern beruht die jetzige Konjunktur des Generationenthemas darauf, dass aktuelle Prognosen und Maßnahmen mit der Erwartung sozialen Fortschritts brechen und damit das sozialstaatliche Entwicklungsmodell gefährdet scheint. ${ }^{30}$

Dass die soziale Sicherung reformiert werden muss, um sie zu bewahren, ist historisch banal. Entscheidend ist, ob die jeweiligen Reformen tatsächlich auf den Wandel der Arbeitswelt und der sozialen Risiken reagieren und dem Bedarf der Menschen an sozialer Sicherheit gerecht werden. Dies darf bezweifelt werden, wenn bloß lineare Kürzungen im bestehenden System mit der Behauptung verbunden werden, es handele sich um langfristig wirksame Strukturreformen.

Insbesondere wäre es ein Missverständnis, dem Sozialstaat anzusinnen, gesellschaftliche Dynamik zu bremsen. Vielmehr geht es darum, sie durch Regulierung und Intervention zu gestalten. ${ }^{31}$ So kann weder rückgängig gemacht noch ignoriert werden, dass immer mehr Frauen erwerbstätig sind und sich Familien verändern. ${ }^{32}$ Der Sozialstaat wird darauf mit substanziell verbesserter Kinderbetreuung und Ganztagsschule reagieren müssen. ${ }^{33}$ Damit können zugleich die ungewollte Kinderlosigkeit und die sozial bedingten Verluste im Bildungssystem für Menschen und Volkswirtschaft vermindert werden. ${ }^{34}$ Unrealistisch wäre es aber, mit dem Sozialstaat demografische Entwicklungen im Einzelnen steuern zu wollen. Seit der Industrialisierung hat sich die demografische Entwicklung nicht linear, sondern dynamisch gestaltet und große Ängste und falsche Prognosen hervorgerufen. Hatte man im 19. Jahrhundert mit Malthus befürchtet, große Teile der Bevölkerung müssten wegen des Bevölkerungswachstums dauerhaft verelenden, so wird seit Beginn des 20. Jahrhunderts vorhergesagt, dass durch eine schrumpfende Bevölkerung die Wirtschaft stagnieren und die Altersversorgung kollabieren werde. Insbesondere in der Wirtschaftskrise der 1920er und 30er Jahre gediehen pessimistische Prognosen. So sagte Friedrich Burgdörfer im Standardwerk für die Bevölkerungspolitik des III. Reiches, »Volk ohne Jugend «, voraus, es werde 2000 nur noch 47 Millionen Deutsche geben und ab 1975 werde die Beitragsbelastung in der Rentenversicherung untragbar werden. ${ }^{35}$ Die Sozialenquête 1966 sagte für 1980 einen »Rentenberg «, für die Zeit ab 2020 eine Entlastung voraus. ${ }^{36} \mathrm{Ob}$ der »Rentenberg « tragbar sein werde, wurde offen gelassen. ${ }^{37}$ In einer dynamischen Gesellschaft ist es auch heute nicht möglich, die demografische Entwicklung oder ihre ökonomischen und sozialen Folgen mit hoher Sicherheit vorherzusagen. ${ }^{38}$

Ruland, DRV 2000, S. 733, 739; Blüm, in: Deufel/Wolf, S. 104, 112.

Dies wird deutlich bei Tremmel, in: SzRG, S. 34, der pauschal unterstellt, dass die bisherigen Generationen Wachstum und Wohlstandszunahme erfahren haben. Dies ist historisch über die Zeit geglättet richtig, abstrahiert aber von aller Individualität, von Krisen und Katastrophen.

Vgl. Becker in: VDR, S. 56, 58 zum Erhalt der Steuerungsfunktion von Recht durch seine Anpassung an gesellschaftliche Veränderungen.

Vgl. Stolleis, FS Zacher, S. 1081, 1093; Geissler, KJ 1997, S. 1, 10.

Vgl. Ruland, FamRZ 2004, S. 493, 500; Scheiwe, S. 147 ff.; Bäcker, in: Butterwegge, S. 244, 259 ff.

Köhler-Rama, DAngVers 2002, S. 449, 450.

Burgdörfer, S. 152 ff., 238; vgl. Tennstedt, in: DRV, S. 51, 54.

Bogs u. a., Sozialenquete-Kommission, S. 165.

Bogs u. a., ebd., S. 168.

$\mathrm{Zu}$ einer umfassenden Kritik an den Annahmen der Bundesregierung und der Hartz-, Herzog- und Rürup-Kommissionen vgl. Bosbach, GMH 2004, S. 96 ff.; Kistler, WSI-Mitteilungen 2004, S. 71, 73 ff.; Eggen, SF 2002, S. 255 ff.; Norbert Blüm weist darauf hin: »Um 1900 hat ein Landwirt drei Nichtlandwirte ernährt. Heute kommen auf einen Landwirt über achtzig Konsumenten. Eigentlich müssten wir nach der Kopfzabltheorie verbungert sein. Wir sind es aber nicht.« In: Deufel/Wolf, S. 104, 110. Die Regierungsfraktionen räumen »Unwägbarkeiten « ein: BT-Drucks. 15/21495om 9. Dezember 2003, S. 18 . 
Auch die in den letzten Jahrzehnten benutzten Prognos-Gutachten müssen laufend revidiert werden. ${ }^{39}$ Darum weist auch der Sozialbeirat der Bundesregierung darauf hin, dass es sich bei Modellrechnungen schon für einen 15-Jahres-Zeitraum nicht um Prognosen, sondern nur um Modelle handeln kann. ${ }^{40}$

Noch weniger kann man annehmen, dass es eine natürliche oder systembedingte demografische Norm gibt. Wenn also der Präsident der Deutschen Gesellschaft für Demographie, Herwig Birg, demografisches Gleichgewicht ohne Wachstum oder Rückgang als erstrebenswert definiert ${ }^{41}$ oder das Bundesverfassungsgericht sich in der Entscheidung zur Pflegeversicherung indirekt für eine Bestandserhaltung stark macht ${ }^{42}$ so ist dies ökonomisch und sozialwissenschaftlich höchst bestreitbar und könnte im Konflikt zu den ökologischen Interessen künftiger Generationen stehen, ${ }^{43}$ ganz sicher ist es aber nicht einem rechtlichen Prinzip zu unterlegen.

Der Sozialstaatsgrundsatz gebietet es, Vorsorgesysteme für die wichtigen Risiken und Lebenslagen zu schaffen, ${ }^{44}$ lässt aber offen, wie Politik und Recht den Lebenslagen in Jugend und Alter gerecht werden und den ökonomischen, sozialen und demografischen Wandel sozial gerecht und akzeptiert gestalten. ${ }^{45}$ Ökonomische Globalisierung und demografische Trends als vorgeblich alternativlose Sachzwänge zu betrachten, heißt, dieser Aufgabe auszuweichen. Damit werden das soziale Staatsziel und seine positive Wirkung auf gesellschaftliche Integration und ökonomische Prosperität gefährdet.

\section{Rechtsstaat}

Das soziale Staatsziel ist in Art. 20 GG mit dem Rechtsstaat verknüpft. Der Rechtsstaat steht dafür, dass erworbene Rechtspositionen geschützt und staatliche Eingriffe in die Gesellschaft begrenzt werden. Wurde deswegen in den ersten Jahren des Grundgesetzes behauptet, Rechtsstaat und Sozialstaat stünden in einem Spannungsverhältnis, so ist im sozialen Rechtsstaat eine Synthese gefunden. ${ }^{46}$ Das soziale Staatsziel wird in ihm nicht kampagnenhaft und willkürlich, sondern berechenbar und rechtsförmig verfolgt. Dies drückt sich in Deutschland vor allem im System des Sozialrechts und in der Sozialversicherung aus. ${ }^{47}$ Die Rentenreformen zumindest bis 1992 drücken das Streben aus, die Dynamik und Zukunftsoffenheit des sozial gestaltenden Staates mit Vertrauensschutz und Verlässlichkeit zu verbinden, die die Menschen für ihre Alterssicherung anstreben. Darin erscheint wieder der doppelte Charakter des Generationenbegriffs: Es geht um Sicherheit für die heute junge Generation, im Alter Ansprüche zu haben, und um den Schutz der späteren Generationen davor, durch diese Ansprüche überfordert zu werden. Der soziale Rechtsstaat kann dabei die Zeitdimension, den Einfluss des Politischen und Gesellschaftlichen nicht leugnen. Es wäre darum wohl besser die Metapher des Generationenvertrages durch die der Generationensolidarität zu

Für die neuesten Prognosen: Römer, SozSich 2003, S. 188 ff.

Gutachten des Sozialbeirats zum Rentenversicherungsbericht 2003 der Bundesregierung, Ziffer 22.

Birg, APuZ 20/2003, S. 6.

BVerfG vom 3. April 2001, BVerfGE 103, 242, 268.

Eggen, SF 2002, S. 255, 258.

Ebsen, in: HS-RV, $\$ 4$, Rz. 32.

Vgl. BVerfG vom 18. Juli 1967, BVerfGE 22, 180, 204; Ebsen, in: HS-RV, $\$ 4$ Rz. 36.

Vgl. Kingreen, S. 121 ff.; Schulin/tgl, Rz. 21 .

47 Zacher, S. 181: "Recht ust jedenfalls das denkbar wirkungsvolliste Medium gewollter sozialer Stabilität«. 
ersetzen. ${ }^{48}$ Deren Einhaltung ist aber stets mit der Erwartung verbunden, sie selbst $\mathrm{zu}$ erhalten. ${ }^{49}$

Das System des SGB VI aus fest erworbenen und als Eigentum geschützten Entgeltpunkten und einem dynamischen, an die Wirtschaftsentwicklung gekoppelten Rentenwert ${ }^{50}$ bringt genau diese Art der Generationengerechtigkeit zum Ausdruck. Da keine Rentenformel allen künftigen Veränderungen gerecht werden kann, wäre es aber ein überzogener Schutz erworbener Rechte, sie von jeder Änderung freizustellen. Nötig ist aber, das Sicherungsziel so zu beschreiben, dass sich der Versicherte langfristig darauf verlassen kann. Ein gesetzliches Ziel existiert allerdings nur in Form einer Berichts- und Prüfungspflicht, ${ }^{51}$ deren Beibehaltung und Ausgestaltung umstritten war. Die jüngsten Reformen haben alle drei Säulen einer dem sozialen Rechtsstaat angemessenen Alterssicherung bedroht: Mit der Streichung zugesagter Entgeltpunkte für Ausbildungszeiten ${ }^{52}$ wird Vertrauen in erworbene Rechte enttäuscht. Wenn Rentenwert und Wohlstandsentwicklung immer weiter entkoppelt werden, ${ }^{53}$ wird das rechtsstaatliche System immer mehr zur Rente nach politisch definierter Kassenlage. Und ein offizieller Abschied vom Sicherungsziel hätte bedeutet, dass das langfristige Vertrauen in ein System, das erhebliche Beiträge verlangt, weiter untergraben würde.

Der Rechtsstaatsgrundsatz verlangt vom Gesetzgeber Respekt vor einmal begründeten Rechtspositionen, in die nicht ohne sachlichen Grund und nicht unverhältnismäßig eingegriffen werden darf. ${ }^{54}$ Der Kampagnencharakter permanenter Reformen auf Zuruf ist damit kaum zu vereinbaren.

\section{Demokratie}

Das dritte Staatsprinzip aus Art. 20 GG ist die Demokratie. Wie das soziale Staatsziel steht sie dafür, dass die Gesellschaft mit ihrer Dynamik Zweck und Richtung von Staat und Recht bestimmt. Demokratie bedeutet das Recht der heutigen Generation, für künftige Generationen zu entscheiden, und das Recht künftiger Generationen, sich davon wieder zu lösen, und steht schon darum einer weitgehenden Gleichbehandlung der Generationen entgegen. ${ }^{55}$ Demokratische Generationengerechtigkeit muss zukünftige Entscheidungen offen halten. ${ }^{56}$ Dass die Vorherrschaft der jeweiligen Gegenwart über die übrige Zeit Schranken benötigt, wird im Umweltstaatsgrundsatz für zukünftige Interessen und im Rechtsstaatsgrundsatz für in der Vergangenheit begründete Rechte ausgedrückt. ${ }^{57}$ Für die Erfüllung des Umweltstaatsgrundsatzes erscheint es notwendig, sachverständige Instanzen zu schaffen, welche die Interessen künftiger Generationen näherungsweise ermitteln und vertreten könnten. ${ }^{58}$

Zacher, S. 518 f.; Ruland, FamRZ 2004, S. 493, 496; Ruland, DRV 2000, S. 733, 751; aus christlich-theologischer Sicht: Kähler, in: VDR, S. 32, 36.

Norbert Blüm erinnert zu Recht an die Formulierung des vierten Gebots: "Du sollst Vater und Mutter ehren, auf dass es dir wohl ergehe und du lange lebest auf Erden. «, (2. Mose, 20; 5. Mose 5, 16), in: Deufel/ Wolf, S. 104, 105. Dazu auch: Kähler, in: VDR, S. 32, 34 ff.

\S 63-68 SGB VI.

$\int 154$ SGB VI.

Änderung von $₫ 74$ SGB VI.

Änderung der Rentenanpassung in $\$ 68$ SGB VI durch Einfügung eines »Nachhaltigkeitsfaktors«.

Bogs, in: FS Zacher, S. 65, 67.

So auch Lux-Wesener, in: SzRG, S. 428.

Nullmeier, in: VDR, S. 65, 73.

Vgl. Häberle, FS Zacher, S. 215, 233.

Kloepfer, DVB1. 1996, S. 73, 78 . 
Die oftmals beklagte Kurzsichtigkeit demokratischer Willensbildung ${ }^{59}$ kann aber auch für den sozialstaatlichen Generationenausgleich zu Über-, Unter- und Fehlausprägungen führen. Betrachtet man die Generationen als Lebenslagen, so ist die aktive Generation gegenüber der nicht wahlberechtigten Jugend und dem immer noch minoritären Alter im Vorteil. Sieht man sie als Kohorten, so besteht die Gefahr, dass die heute aktive Generation sich heute aus kurzfristigen Erwägungen nicht belasten will - etwa durch höhere Rentenbeiträge oder Steuern -, obwohl ihr dies durch höhere Rentenansprüche und eine besser ausgebildete nachrückende Generation in Zukunft zugute käme. ${ }^{60}$

Probates Mittel gegen demokratische Fehlentscheidungen ist, den ihnen vorangehenden Diskurs zu verbessern. Dass der demokratische Diskurs gefährdet ist, kann vermutet werden, wenn nicht über Alternativen diskutiert wird, sondern behauptet werden kann, es gebe keine Alternative ${ }^{61}$ In einem idealen und rationalen Diskurs sind möglichst alle Interessen und Informationen präsent, und es kann ohne verzerrende Ängste und Druck über Alternativen entschieden werden. Im rentenpolitischen Diskurs dominieren jedoch nicht nur die Interessen der heute aktiven Generation über die heute zu unterstützenden Generationen, sondern auch die der heute aktiven Kohorte über ihre eigenen Interessen im Alter. So wird systematisch nicht wahrgenommen, dass die heute beschlossenen Eingriffe in die Rentenanpassung nicht vorrangig die heutigen Rentner, sondern vor allem die heute aktive und die ihr nachfolgenden Generationen betreffen, ${ }^{62}$ weil der heutige Rentenwert Basis für den künftigen ist. Dies ist zwar durchaus präsent, indem viele ihre Alterssicherung als gefährdet betrachten. Dies wird jedoch nicht selbst verantwortet, wie es demokratisch wäre, sondern anonymen Mächten zugerechnet wie Demografie, Globalisierung oder gar der heute zum Teil noch nicht geborenen Generation der aktiven Beschäftigten von 2030, der in $\$ 154$ Abs. 3 Nr. 1 SGB VI unterstellt wird, sie sei nicht bereit, einen $22 \%$ übersteigenden Rentenbeitrag zu zahlen. Das liegt auch daran, dass dynamische Entwicklungen über lange Zeit schwer vorherzusagen sind. Menschen sind zumeist nur in der Lage, lineare Trends fortzuschreiben, ${ }^{63}$ was erklärt, dass in jeder Wirtschaftskrise auch langfristig alles düster erscheint. ${ }^{64}$ Dazu kommt, dass sich stets Experten finden, die aus gegenwärtigen Interessen längerfristige Prognosen abgeben. Angesichts des Mangels an langfristiger Vorstellungskraft fehlt das Korrektiv eigener Interessen und Erfahrung, das im Diskurs bei anderen Themen gegen die Expertensicht wirksam wird. Ein zweites Mittel, um zu fördern, dass schwierige Themen sachgerecht und demokratisch entschieden werden, ist die dezentrale Selbstverwaltung der betroffenen Personen, damit diese besondere Sachnähe und Sachkunde nutzen und entwickeln können. So wird die gesetzliche Rentenversicherung paritätisch von Gewerkschaften und Arbeitgebern verwaltet. Da die Selbstverwaltung jedoch nicht

60 Dieses Problem ist für die individuelle Alterssicherung als »gegenwartsverzerrte Präferenz« bekannt, vgl. Leinert, SF 2004, S. 55, ff.

61 BT-Drucks. 15/2149, S. 3; die floskelhafte Behauptung in Gesetzentwürfen ist inzwischen in den politischen Argumentationshaushalt übernommen worden.

62 Deml, SozSich 2002, S. 411, 418; differenziert: Nullmeier, KJ 1997, S. 261, 266; dies wird etwa übersehen von Hebeler, DRV 2002, S. 270, 280; vgl. Jaeger, NZS 2003, S. 225, 226, die zutreffend darauf hinweist, dass für die Sicherungslücken der heute aktiven Generation die dann nachfolgende Generation durch Unterhalt oder über Steuermittel aufkommen muss.

63 Bosbach, GMH 2004, S. 96, 98: "50-Jahres-Prognosen sind moderne Kaffeesatzleserei«.

64 Dagegen wird der Geburtenrückgang z. B. in einer Untersuchung aus dem Jahr 1971 als wirtschaftlich eher anregend bewertet. Es werden nur wenige Probleme gesehen: »Es wird nötig sein (..) der Ausbildung und Umschulung der Arbeitskräfte größere Aufmerksamkeit zu geben (..). Vor allem gilt es auch die psychologischen Vorurteile gegenüber einem Bevölkerungsstillstand abzubauen, da sie die wirtschaftliche Aktivität beeinträchtigen.", Wander, S. 15. 
über Beitragssatz und Rentenansprüche entscheiden kann und ihre Argumente bei

Rentenreformen nur wenig gehört werden, ist dieses Korrektiv kaum entwickelt. Es bleibt festzuhalten, dass die demokratische Entscheidung über den Ausgleich der Generationen für Defizite des demokratischen Diskurses anfällig ist. Experten, die den Eindruck erwecken, sicheres Wissen über langfristige Entwicklungen zu besitzen, helfen diesen Defiziten nicht ab, sondern verstärken sie.

\section{Schutz der Familie}

Besondere Verfassungsnorm zum Generationenverhältnis ist der Schutz von Ehe und Familie nach Art. 6 GG. Das vorgefundene gesellschaftliche Institut, das Generationen hervorbringt und verbindet, ist besonders geschützt. Wurde dieser Schutz zunächst vor allem als Garantie einer von staatlichen Eingriffen freien Privatsphäre verstanden, hat das Bundesverfassungsgericht vor allem im letzten Jahrzehnt aus Art. 6 GG eigene Vorgaben für die Familien- und Rentenpolitik entwickelt.

Dies knüpft an der Erkenntnis an, dass Art. 6 GG ein besonderer Gleichheitssatz ist, der dem Gesetzgeber verbietet, Ehe und Familie zu benachteiligen. ${ }^{65}$ In vielen Konstellationen ist das unproblematisch. ${ }^{66}$ Der Teufel sitzt jedoch im Detail. $\mathrm{Zu}$ bestimmen ist nämlich erstens, wann eine zu schützende Familie vorliegt, zweitens, wann sie benachteiligt wird. Da jeder Mensch aus einer Familie hervorgeht und familienrechtlich nicht nur mit möglichen Kindern, sondern auch mit seinen Eltern lebenslang durch Beistands- und Unterhaltspflichten verbunden ist, ist es nicht leicht, Familien von Nicht-Familien abzugrenzen. ${ }^{67}$ Die griffige Formel »Familie ist, wo Kinder erzogen werden « taugt für Fortschritt gegenüber einem ehezentrierten Familienmodell und Abbau ungerechtfertigter Ungleichbehandlungen, ${ }^{68}$ erfasst aber nicht alle Konstellationen familiären Generationenausgleichs. So hat das Bundesverfassungsgericht es für richtig gehalten, dass Personen, die Kinder erziehen, bei der Beitragsbemessung zur Pflegeversicherung begünstigt werden sollen, ${ }^{69}$ und hat dem Gesetzgeber aufgetragen, dies auch für die Rentenversicherung zu prüfen. ${ }^{70}$ Der Bundesrat hat sich die Forderung nach Berücksichtigung von Kindern bei den Rentenbeiträgen zu eigen gemacht. ${ }^{71}$ Für die Pflegeversicherung wurde diskutiert, ob dieser Bonus nur aktuell erziehenden Eltern oder auch den erzogen habenden Eltern zugute kommen soll (was dann fast $80 \%$ der Bevölkerung wären). ${ }^{72}$ Schon das zeigt die Schwierigkeiten. ${ }^{73}$ Soll aber eine kinderlose Person diese Regel für gerecht halten, wenn sie möglicherweise jahrelang ihre eigenen Eltern gepflegt und unterstützt hat?

Noch schwieriger ist zu definieren, wann eigentlich die Familie benachteiligt wird. Man kann mit dem Bundesverfassungsgericht die Familien benachteiligt sehen, weil Eltern für gleiche Beiträge das Recht auf eine Rente oder Pflegeleistungen BVerfG vom 17. Januar 1957, BVerfGE 6, 55, 76.

66 Vgl. BVerfG vom 27. Mai 1970, BVerfGE 28, 324, 347 (Heiratsklauseln zum Ausschluss der Waisenrente).

67 Vgl. BVerfG vom 18. Juni 1975, BVerfGE 40, 121, 132 zum Ende der Waisenrente auch für behinderte Menschen mit 25 Jahren.

68 So BVerfG vom 29. Oktober 2002, BVerfGE 106, 166, 175, 179 im Kindergeldrecht.

69 BVerfGE 103, 242, 257.

70 BVerfGE 103, 242, 270.

71 BT-Drucks. 15/2562 vom 26. Februar 2004, S. 8 (Stellungnahme zum RV-Nachhaltigkeitsgesetz).

72 Vgl. 15. WP, 89. Sitzung des Bundestages: MdB Horst Seehofer, Prot, S. 7943; BM Ulla Schmidt, S. 7945; MdB Annette Widmann-Mauz, S. 7958 f.

73 Diese liegen schon in der Sachverhaltsanalyse durch das BVerfG begrundet, wie Ruland, NJW 2001, S. 1673, 1674, darstellt. 
erwerben wie Kinderlose, obwohl sie viel für ihre Kinder aufwenden und nur diese in der Zukunft Renten- und Pflegeversicherungsbeiträge zahlen können, aus denen die Renten und Pflegeleistungen der Kinderlosen wie der Eltern bestritten werden. ${ }^{74}$ Andererseits gibt es diesen Effekt bei jeder kollektiven Alterssicherung, weil sie gerade gegen das Risiko versichert, keine oder keine leistungsfähigen Kinder zu haben, die einen im Alter unterstützen könnten. ${ }^{75}$ Hält man es für sozial ungerecht, dass Eltern im Generationenausgleich doppelt belastet sind, gibt es mindestens drei Lösungen: Der zuletzt vom Bundesverfassungsgericht nahegelegte Beitragsbonus erscheint keineswegs als zwingend. Kinder werden so als »Naturalbeitrag « zur Pflegeund Rentenversicherung gewertet, auch wenn sie niemals in diese einzahlen. Dass nicht nur die Kinder als Beitragszahler von morgen, ${ }^{76}$ sondern auch das produktive Wissen und die Bildung, ${ }^{77}$ das Realkapital und die Ersparnis von morgen heute vorbereitet werden ${ }^{78}$ - auch von Kinderlosen und auch dies Voraussetzungen für die Alterssicherung von morgen sind, bleibt unberücksichtigt. ${ }^{79}$ Das Bundesverfassungsgericht meint, die Voraussetzungen der sozialen Alterssicherung auf Beiträge und Kinder abstrahieren zu können. ${ }^{80}$ Dazu kommt, dass das Bundesverfassungsgericht den Zusammenhang zwischen Kapital und Arbeit zerreißt, wenn es glaubt, dass private Systeme der Pflege- oder Alterssicherung nicht auf nachwachsende Generationen angewiesen seien. ${ }^{81}$ Tatsache ist, dass jeder Sozialaufwand - ob von Sozialversicherung, Privatversicherung oder Privatpersonen gedeckt - immer aus aktueller Wertschöpfung gedeckt werden muss und darum auf alle Produktionsfaktoren angewiesen ist. ${ }^{82}$ Eine Alterssicherung »für sich selbst « ohne andere Wirtschaftssubjekte und eine nachrückende Generation ist in keinem System möglich. Es kommt nur darauf an, in welchen politischen und rechtlichen Formen der Zusammenhang der Generationen vermittelt wird. Wirtschaftlich, aber auch rechtlich sind die alten Menschen in jedem System und jeder Rechtsform der Altersvorsorge auf einen Träger, Vertragspartner oder Unterhaltsverpflichteten angewiesen, der aktuell leistungsfähig ist. Bestünde die Altersvorsorge nur aus Geld, so müsste dessen Kaufkraft durch eine Zentralbank und eine funktionierende Volkswirtschaft gesichert sein. Gespartes Geld kann man nicht essen. Für eine gewisse Zeit ist nur das abbezahlte Eigenheim eine Ausnahme von dieser Regel - aber nur solange, wie der alte Mensch nicht pflegebedürftig oder das Haus renovierungsbedürftig wird.

Rentensteigernde Kindererziehungszeiten ${ }^{83}$ - als Folge der verfassungsgerichtlichen Rechtsprechung der neunziger Jahre - sind ein Kompromiss zwischen der Theorie vom Naturalbeitrag und einer Kompensation erziehungsbedingter Siche-

bericht 2003 der Bundesregierung, Ziffer 57.

76 BVerfGE 103, 242, 264.

77 Dieses Argument für die Anerkennung von Ausbildungszeiten wird heute oft übersehen, vgl. Blüggel, SozSich 2004, S. 61, 65; Nullmeier, KJ 1997, S. 261, 276; zur Kontroverse in der Gesetzgebung: BTDrucks. 15/2562 vom 26. Februar 2004, S. 8; BT-Drucks. 15/2591 vom 3. März 2004, S. 2 f.

78 Schmähl, DAngVers 2003, S. 349, 362; Köhler-Rama, DAngVers 2002, S. 449, 450; Ebert, in: Butterwegge/Klundt, S. 99, 104.

79 Nach BVerfGE 94, 241, 264 dürfen weitere Zusammenhänge ausgeblendet werden.

80 BVerfGE 94, 241, 263; 87, 1, 40.

81 BVerfG vom 3. April 2001, BVerfGE 103, 271, 292 zur Ungleichbehandlung von gesetzlich und privat Pflegeversicherten.

82 Ruland, FamRZ 2004, S. 493, 496; Ruland, NJW 2001, S. 1673, 1676; Mackenroth, S. 23 f.; Zacher, S. 195 f.; Reuter, in: Butterwegge/Klundt, S. 81, 90 ff.; Blüm, in: Deufel/Wolf, S. 104, 109; Zacher, S. 181, schreibt: "Kein Rentengesetz kann, wenn die Wirtschaft sie nicht erbringt, die Mittel schaffen, die notwendig sind, um die Renten zu zablen. " Dieser Satz gilt auch, wenn Rentenzahlung von privatrechtlichen Institutionen beansprucht wird und diese nicht mehr leistungsfähig sind. Vgl. zur Diskussion über Umlageverfahren oder Kapitaldeckung unter diesem Aspekt bei der Schaffung der Rentenversicherung: Tennstedt, in: VDR, S. $51 \mathrm{ff}$. und den Bezug aul Miathäus 6,34. 
rungslücken der Eltern, zumal wenn sie nach verfassungsgerichtlicher Rechtsprechung unabhängig davon zu gewähren sind, ob in den Erziehungszeiten versicherungspflichtig gearbeitet wurde oder nicht. ${ }^{84}$

Es wäre schließlich auch möglich, Kinderbetreuung und Schulwesen so zu verbessern, dass erziehungsbedingte Sicherungslücken und ungewollte Kinderlosigkeit nicht entstehen müssten, weil Kindererziehung und Erwerbstätigkeit vereinbar sind. ${ }^{85}$ Dies würde aber einen Mitteleinsatz außerhalb des Rentenversicherungssystems voraussetzen und würde daher den verfassungsgerichtlichen Vorgaben ${ }^{86}$ nicht gerecht werden, obwohl das Bundesverfassungsgericht bei anderer Gelegenheit ausgeführt hat, dass der Staat zum Schutz des ungeborenen Lebens, von Ehe und Familie und zur Gleichstellung der Geschlechter verpflichtet ist, die Grundlagen dafür zu schaffen, dass Familie und Erwerbstätigkeit aufeinander abgestimmt werden. ${ }^{87}$

Daraus folgt: Das Bundesverfassungsgericht hat ohne zwingenden Grund im Text des Grundgesetzes sich in einer familien- und sozialpolitischen Kontroverse positioniert und diese faktisch entschieden, ${ }^{88}$ indem eine Möglichkeit als verfassungsrechtlicher Handlungsauftrag privilegiert wurde. Damit ist es abgeschnitten worden, offen demokratisch zu entscheiden, wie Familien sozialstaatlich unterstützt werden sollen. So hat das Verfassungsgericht dem Ausgleich zwischen den Generationen trotz guten Willens nicht geholfen.

\section{Sicherung des Existenzminimums}

Das Existenzminimum durch staatliche Fürsorge zu jedem Zeitpunkt des Lebenslaufs - und damit insbesondere in Kindheit und Alter - zu sichern, war der erste Schritt zum sozialstaatlichen Generationenverhältnis. Im sozialen Rechtsstaat ist daraus ein Anspruch auf Sozialhilfe und Grundsicherung im Alter geworden. ${ }^{89}$ Letzterer ist im Regelfall nicht mehr nachrangig zum Unterhaltsanspruch gegen die Kinder. ${ }^{90}$

Das Existenzminimum ist ein zweiter Ansatzpunkt für die Rechtsprechung des Bundesverfassungsgerichts zum Familienleistungsausgleich. Aus der Garantie des Existenzminimums hat das Gericht gefolgert, dass dieses nicht besteuert werden darf. ${ }^{91}$ Weiter wurde vom Gericht geschlossen, dass dies für jeden mit unterhaltenen Familienangehörigen gelten müsse, um Familien nicht zu benachteiligen. ${ }^{92}$ Als Freibetrag muss dieses Existenzminimum auch bei dem verschont werden, der weit mehr als das Minimum zu versteuern hat. ${ }^{93}$ Der Gesetzgeber hat darauf reagiert und das Kindergeld ins Steuerrecht integriert. Man darf nun zwischen Kindergeld und Kinderfreibetrag wählen. Damit soll das Existenzminimum des Kindes gesichert sein. Die staatliche Leistung beträgt für jedes erste bis dritte Kind 154 Euro, für jedes weitere Kind 179 Euro. ${ }^{94}$ Ist ein jährlicher Steuerfreibetrag von

BVerfG vom 12. März 1996, BVerfGE 94, 241, 260.

Zu diesem Ziel im rentenrechtlichen Kontext: BT-Drucks. 15/2591 vom 3. März 2004, S. 4 f.; BTDrucks. 15/2149 vom 9. Dezember 2003, S. 19; Ruland, DRV 2000, 733, 737.

Noch vorsichtig: BVerfGE 87, 1, 39; dagegen setzt BVerfGE 103, 242 bereits voraus, dass die Lösung der Probleme nur innerhalb des Sozialversicherungsrechts erfolgen könne; kritisch dazu: Haass, KJ 2002, S. 104, $105 \mathrm{ff}$.

BVerfG vom 28. Mai 1993, BVerfGE 88, 203, 260 f.; bekräftigt in BVerfGE 99, 216, 234.

Ähnlich: Eichenhofer, MittLVA Ober- und Mittelfranken 2001, S. 719, 722.

BVerwGE 1, 159; Papier, S. 859.

\2 Abs. 1 Satz 3 Grundsicherungsgesetz (GSiG).

BVerfG vom 29. Mai 1990, BVerfGE 82, 60, 85; BVerfG vom 25. September 1992, BVerfGE 87, 153, 169.

BVerfGE 82, 60, 86; 87, 153, 169; 99, 216, 233.

BVerfGE 99, 216, 233; 99, 246, 263; kritisch: Sacksofsky, NJW 2000, S. $1896,1902$.

\66 EStG. 
5808 Euro $^{95}$ günstiger als das Kindergeld, wird dieser gewährt. ${ }^{96}$ Damit werden alle Familien mit Kindern unabhängig vom Bedarf gleich gefördert - außer den ca. $5 \%$ mit dem höchsten Einkommen, die mehr erhalten. ${ }^{97}$ Die staatlichen Transfers zur jungen Generation folgen nicht der Bedarfsgerechtigkeit, sondern der Besitzstandsgerechtigkeit.

Die verfassungsgerichtliche Rechtsprechung blockiert nicht nur eine Verschiebung des Kinderausgleichs im Rentenrecht auf die Leistungsseite im Alter, ${ }^{98}$ sondern durch seine Fixierung auf monetäre Transfers im Steuer- oder Beitragsrecht auch einen Einsatz der Mittel für die soziale Infrastruktur. Dass dieses System verfassungsrechtlich geboten sein soll und es dem Gesetzgeber also verboten sein sollte, die für Steuer- und Beitragsentlastungen aufgewandten erheblichen Mittel alternativ für bessere und erschwingliche Kinderbetreuung und Ganztagsschulen auszugeben, ${ }^{99}$ ist schwer zu glauben, wird sich aber solange nicht ändern, wie keine politische Kraft öffentlich kritisiert, dass das Verfassungsgericht tief in die Politikgestaltung eingegriffen hat ${ }^{100}$ und die rechtliche Kritik an der Bindungswirkung familienpolitischer Bekenntnisse ${ }^{101}$ vereinzelt bleibt. Bis dahin ist bei der Förderung der Familien ein System entstanden, das sich mit guten Gründen als sozial ungerecht kritisieren lässt, weil Steuer- und Beitragsentlastungen einem Teil der Kinder gar nicht zugute kommen und vor allem die förderungsbedürftigsten Kinder nicht erreichen können. Dieses System trägt dazu bei, dass die für die Zukunft nötige gezielte Förderung der Eltern und der jungen Generation ${ }^{102}$ unterbleibt und Deutschland auf diesem Sektor europäische Standards nicht erreichen kann. ${ }^{103}$

\section{Eigentum und Erbrecht}

Generationengerechtigkeit kann auch an dem in Art. 14 Abs. 1 GG gewährleisteten Eigentum und Erbrecht anknüpfen. Dem, der hinreichend Eigentum hat und erbt, ermöglicht es, im Alter gesichert zu leben. Mit dem Erbrecht ist verbunden, dass in einer Generation erworbene soziale Unterschiede sich in der nächsten Generation von Anfang an fortsetzen. Dies kann in der vererbenden Generation einen Leistungsanreiz schaffen, kann aber in der erbenden Generation leistungsfeindlich wirken und gesellschaftliche Dynamik hemmen. Wichtiger als individuelle Erbschaften kann es sein, der nachfolgenden Generation viel auf der Ebene kollektiv geteilter Güter wie Naturschätze, Infrastruktur, Kultur und Bildung zu hinterlassen. ${ }^{104}$ Heutige Investitionen in kollektive Güter können ein wichtigerer Beitrag

95 Im Regelfall, $₫ 32$ Abs. 6 Satz 1 und 2 EStG.

$96 \rrbracket 31$ Satz 4 EStG.

97 Vgl. Bäcker, in: Butterwegge, S. 244, $256 \mathrm{f}$.

98 Nur darauf stellt Lenze, NZS 2003, S. 505, 511 ab, wenn sie das Konzept des BVerfG als positiv für innergenerationelle Gerechtigkeit wertet.

99 Nach BVerfGE 82, 60, 88 ist die staatliche Finanzierung des Schul-, Bildungs- und Ausbildungssystems für die gebotene Steuerverschonung der Familien »ohne Bedeutung «; in BVerfGE 99, 216, 234 erscheint die »Fremdbetreuung « als zu tolerierende Besonderheit.

100 Diese Absicht und das zu Grunde liegende konservative Familienbild sind durchaus offen gelegt, vgl. Kirchhof, NJW 1996, S. 1497, 1502 f.

101 Sacksofsky, NJW 2000, S. 1896, 2002, zu BVerfGE 99, 216; Ruland, NJW 2001, S. 1673, 1676 zur »verfassungskonformen Auslegung « von BVerfGE 103, 242; Haass, KJ 2002, S. 104, 110 zur Problematik für das offene demokratische Verfahren.

102 Vgl. Scheiwe, S. 323 ff.; Bäcker, in: Butterwegge, S. 244 ff; Lange/Lauterbach/Becker, in: Butterwegge/ Klundt, S. 153 ff., zum Zusammenhang von Armut und Bildungschancen.

103 Dies haben auch die Regierungsfraktionen erkannt, BT-Drucks. 15/2580 vom 3. März 2004, S. 2; vgl. Kaufmann, S. 290, $303 \mathrm{f}$.

104 Vgl. dazu den Vorschlag von Broß, JZ 2003, 3. 874, 878; undifferenziert für eine als gerecht empfundene hohe Sparrate aber Lumer, in: SzRG, S. 105, 115. 
zum Wohlergehen der kommenden Generationen sein als das Hinterlassen von Geld. ${ }^{105}$ Wichtig wäre es, dass der Sozialstaat in Kindheit und Jugend vor allem die nicht und wenig erbenden Teile einer Generation fördert und sich auf die eigene Leistung fordernde und ermöglichende Bildung konzentriert. Die soziale und demografische Entwicklung führt zu einer immer stärkeren Ungleichverteilung ererbten Vermögens, was nahe legen würde zu überprüfen, in welchem Ausmaß Erbschaften zur Finanzierung bislang defizitärer kollektiver Vorsorge herangezogen werden. Dies geschieht derzeit nicht.

Für die Alterssicherung wichtig ist, dass nach der Rechtsprechung des Bundesverfassungsgerichts Rentenanwartschaften als Eigentum zu schützen sind. ${ }^{106} \mathrm{Da}-$ mit hat der Vertrauensschutz eine Anknüpfung. Das Gericht begründet und konkretisiert dies so, dass Rentenanwartschaften auf eigener Leistung beruhten, wie Eigentum der Freiheits- und Existenzsicherung dienten und den Versicherten als persönliches Recht zugeordnet sind. ${ }^{107}$ Der Eigentumsschutz von Anwartschaften hat sich jedoch bislang nicht als starke Schranke von Rentenreformen erwiesen. Dem Umfang nach ist der Schutz beschränkt, indem nur auf Eigenleistung beruhende Anwartschaften voll umfasst sein sollen. ${ }^{108}$ Damit sind Kürzungen der Anwartschaften für Hinterbliebene ${ }^{109}$ oder der Zusagen für Ausbildungszeiten ${ }^{110}$ schwächer geschützt. Dies erscheint nicht zwingend: Das Eigentum an privatem Vermögen zur Alterssicherung muss nicht auf Eigenleistung beruhen und auch nicht a priori der Existenzsicherung dienen. ${ }^{111}$ Es kann auch ererbt oder geschenkt sein. Es ist nur geschützt, weil es bereits bestanden hat. Für die meisten Sozialversicherten haben hingegen Anwartschaften auch dann wichtige existenz- und freiheitssichernde Funktion, wenn sie aus anderen Gründen als eigener Geldleistung zugesagt wurden. ${ }^{112}$ So einleuchtend es ist, dass nicht jede in der Vergangenheit zugesagte staatliche Leistung Eigentumsschutz gewinnen kann, so sehr ist es für langfristige Ansprüche auf Alterssicherung zweitrangig, aus welchem Grund sie zugesagt wurden. Dies zeigt, dass hier Vertrauensschutz und Sozialstaatsgebot mindestens gleichrangige verfassungsrechtliche Argumente für ein Recht auf gesicherte Teilhabe im Alter sein müssen. ${ }^{113}$ Im sozialrechtlichen Umlageverfahren ist es ohnehin so, dass Entgeltpunkte nicht Anrechte auf Rückzahlung eingezahlten Geldes sind, ${ }^{114}$ sondern Anrechte auf künftige Leistung und Teilhabe am gesellschaftlichen Reichtum. ${ }^{115}$ Mit dem wachsenden Gewicht der Steuerfinanzierung und etwa der für Kindererziehung erworbenen Entgeltpunkte ist zu fragen,

Deml, SozSich 2002, S. 411, 418; dies ist auch bei der Diskussion um Art. 115 Abs. 2 Satz 2 GG und kreditfinanzierte Investitionen zu bedenken, zu der hier aus Platzgründen nicht weiter Stellung bezogen wird.

106 BVerfG vom 28. April 1999, BVerfGE 100, 1, 32 (Zusatz- und Sonderversorgungssysteme der DDR); BVerfG vom 8. April 1987, BVerfGE 75, 78, 96 f. (Berufs- und Erwerbsunfähigkeitsrenten); BVerfG vom 4. Juni 1985, BVerfGE 70, 101, 110; BVerfG vom 16. Juli 1985, BVerfGE 69, 272, 298; BVerfG vom 10. Mai 1983, BVerfGE 64, 87, 97; BVerfG vom 1. Juli 1981, BVerfGE 58, 81, 109; BVerfG vom 28. Februar 1980, BVerfGE 53, 257, 289; dazu Neumann, DRV 1999, S. $393 \mathrm{ff}$. BVerfGE 100, 1, 33; 69, 272, 300; 53, 257, 292

108 Dazu auch Papier, S. 865 f.

109 BVerfG vom 18. Februar 1998, BVerfGE 97, 271, 283.

110 BVerfGE $58,81,112$.

111 Lenze, SF 2002, S. 33, 37.

112 Bieback, KJ 1998, S. 162, 170.

113 Vgl. die Differenzierung des BSG, SozSich 2003, S. 207, 210 f; dazu Lenze, NZS 2003, S. 505 ff.; vgl. auch die abweichende Meinung der Richter Benda und Katzenstein zur Beschränkung von Ausbildungszeiten nach dem Zwanzigsten Rentenanpassungsgesetz von 1977, BVerfGE 58, 129; Lenze, SF 2002, S. 33, 35, 39; Bogs, FS Zacher, S. 65, 70 ff.; Stolleis, FS Zacher, S. 1081, 1097; Bieback, KJ 1998, S. 162, $171 \mathrm{ff}$. für das Sozialstaatsgebot; Schlenker, S. 154, 252 f.; Becker, in: VDR, S. 56, 63, Papier, S. 868; Blüggel, SozSich 2004, S. 61, 68 für den Vertrauensschutz.

114 Vgl. Schlenker, S. 154 ff.; es kann daher nicht zentral auf die wie immer berechnete »Eigenfinanzierungsquote « ankommen, wie Papier, $\mathrm{S} .860 \mathrm{f}$., meint. 
ob auch diese nicht geschützt sind oder ob alle Punkte in Höhe der Steuerfinanzierungsquote entzogen werden könnten. ${ }^{116}$ Dies könnte kein vernünftiges Ergebnis sein.

Das zweite Problem des Eigentumsschutzes ist, dass er vor allem die Entgeltpunkte, und nur sehr eingeschränkt ihren Wert und dessen Anpassung schützt. Das Bundessozialgericht sieht immerhin den Schutz vor Inflation als geschützt an, nicht jedoch eine Anpassung nach Lohn- und Wirtschaftsentwicklung. ${ }^{117}$ Dies entspricht zwar in gewisser Weise dem Schutz zivilrechtlicher Eigentumstitel, die ebenfalls nicht davor schützen, dass Geld oder Aktien an Wert verlieren. Da der Rentenwert an die Einkommens- und Wirtschaftsentwicklung gekoppelt ist und der Versicherte Chancen und Risiken der Produktivitätsentwicklung mitträgt, kann seine Entwicklung nicht von der Wirtschaftsentwicklung losgelöst sein. ${ }^{118}$ Dass es dem Gesetzgeber frei stehen soll, ob er dieses Ziel über die Koppelung an Bruttolohn, Nettolohn oder Bruttosozialprodukt verwirklicht, sichert einen nötigen Spielraum. Problematisch wird es aber, wenn die Anpassungsfaktoren ständig wechseln oder frei kombiniert werden, Nullrunden eingelegt werden oder der Rentenanstieg durch willkürliche Formeln begrenzt wird, ${ }^{119}$ so dass sich im Ergebnis das Sicherungsniveau auf kaum planbare Weise vermindert. ${ }^{120}$ Der jetzt eingefügte zusätzliche Nachhaltigkeitsfaktor besteht aus der Entwicklung des Verhältnisses von Rentnern zu Erwerbstätigen und einem Gewichtungsfaktor, der am Beitragssatzziel von $22 \%$ im Jahr 2030 orientiert worden ist. ${ }^{121}$ Weil die Gewichtung dieses Faktors allein am gewünschten Beitragssatz orientiert ist, steht diese Größe gerade nicht für eine nachvollziehbare Risikoverteilung zwischen Aktiven und Rentnern. Weiterhin werden seit 2001 die (größtenteils fiktiven) privaten Altersvorsorgeaufwendungen berücksichtigt, was den Rentenanstieg ebenfalls dämpft. ${ }^{122}$

Anders als bei der Aktie ${ }^{123}$ können Rentenversicherte angesichts dieser Eingriffe nicht das System wechseln, für das sie Pflichtbeiträge zahlen. Und anders als bei der Inflation von Geld ist der relative Wertverlust des Rentenwerts kein Schicksal, das die ganze Volkswirtschaft trifft. Dass Anpassungen zumindest vor teuerungsbedingtem Wertverlust schützen sollten, wie es das Bundessozialgericht meint, erscheint aber als notwendige, wenn auch kaum hinreichende untere Haltelinie gegen eine Entwertung des ganzen Systems durch willkürliche Eingriffe. Umso wichtiger sind gesetzliche Regeln, die eine Niveausicherung vorsehen.

\section{Schutz vor unnötiger Zwangsmitgliedschaft}

Ein das Problem der Rentenanpassung treffender Gesichtspunkt ergibt sich beim Rentenwert nicht aus dem Eigentumsschutz, sondern aus dem Schutz vor einer ungerechtfertigten Einbeziehung in ein Pflichtsystem, der sich aus der allgemeinen

Lenze, SF 2002, S. 33, 39.

BSG vom 31. Juli 2002, SozSich 2003, S. 207, 210; das BVerfG hat die Frage skeptisch offen gelassen, BVerfGE 64, 87, 98; 100, 1, 44.

BVerfGE 64, 87, 105; Papier, S. 889.

Zur Kritik daran bereits Schmähl, SF 2001, S. 2 ff.

Nürnberger, SozSich 2003, S. 330, 334.

Vgl. dazu \68 Abs. 5 SGB VI in der Fassung des RV-Nachhaltigkeitsgesetzes: Im Nachhaltigkeitsfaktor ist eine Größe a enthalten, die am Beitragssatzziel orientiert ist, BT-Drucks. 15/2149 vom 9. Dezember 2003, S. 18. Zur Kritik an der Beitragssatzorientierung des so gefassten Nachhaltigkeitsfaktors: Engelen-Kefer/Franz/Schoch/Wiesehügel, S. 50 f. Gegen den Gesetzesbeschluss zum RVNachhaltigkeitsgesetz hatte der Bundesrat in seiner Sitzung vom 14. Mai 2004 Einspruch eingelegt, den der Bundestag am 16. Juni 2004 zurückgewiesen hat.

Vgl. zu der Entwicklung dieses Faktors \$2 25 e Abs. 4 SGB VI.

$\mathrm{Zu}$ diesem Vergleich: Lenze, NZS 2003, S. 506. 

cherung für eine wachsende Anzahl von Versicherten zu einem Anspruch führt, der das als Mindestsicherung im Alter ohnehin zu beanspruchende Existenzminimum ${ }^{124}$ nicht übersteigt, so ist die Pflichtmitgliedschaft nicht zu rechtfertigen. Führt eine typische Erwerbsbiographie nicht mehr zu einem hinreichenden Rentenanspruch oberhalb der Mindestsicherung, so würde das System im Ganzen verfassungswidrig, wie das Bundessozialgericht zutreffend festgestellt hat. ${ }^{125}$ Dieser Punkt ist wohl noch nicht erreicht, rückt aber durch die neuerlichen Reformen näher. ${ }^{126} \mathrm{Ob}$ der kritische Punkt für die real existierenden Rentenversicherten allerdings erkannt werden kann, wenn die Niveausicherungsklausel nur an der Entwicklung der Standardrente nach 45 durchschnittlichen Berufsjahren ansetzt, ${ }^{127}$ muss bezweifelt werden. Schon wird befürchtet, dass erst nach 35 durchschnittlichen Beitragsjahren das Grundsicherungsniveau erreicht wird. ${ }^{128}$ Die Berichtsund Beobachtungspflicht müsste weit umfassender für die Entwicklung der Renten in verschiedenen typischen Erwerbssituationen gelten.

Es ist daran zu erinnern, dass die in der Rentenversicherung pflichtversicherte Mehrheit der Bevölkerung durch Steuern auch den größten Teil der Mindestsicherung und der Beamtenversorgung bezahlt. Als abhängig Beschäftigte erwirtschaften sie schließlich auch zu einem erheblichen Teil die Renditen von Lebensversicherungen und Vermögenstiteln der außerhalb der Sozialversicherung stehenden Personen, während sie selbst nur wenig Vermögen bilden können.

Wenn die heute jüngere Generation ein System finanziert, das den Lebensstandard der meisten heutigen Rentner sichert, der Rentenwert aber bis zum Ruhestand dieser Generation durch neu eingefügte Faktoren nicht mehr mit dem allgemeinen Lebensstandard wächst und größere Teile dieser Generation Renten auf Grundsicherungsniveau erhielten - dann wäre das ein Problem der Gerechtigkeit, ${ }^{129}$ allerdings vor allem innerhalb dieser Generation. ${ }^{130}$ Die Folge könnte eine selbstverstärkende Vertrauenskrise des Sozialstaats sein. ${ }^{131}$ Die heutigen Rentenreformen sind aber nicht Lösung, sondern Ursache dieses Problems. ${ }^{132}$ Denn dass sich die Alterssicherung der heute jungen Generation vom Schicksal der Volkswirtschaft abkoppelt, wäre weder im Guten noch im Bösen zu befürchten, wenn es bei einer transparenten lohnorientierten Formel der Rentenanpassung bliebe oder die Rentenentwicklung in Zukunft an das Wachstum des Volkseinkommens angeknüpft würde.

Die Riester-Förderung privater und betrieblicher Vorsorge ${ }^{133}$ vermag dieses Problem nicht zu kompensieren. Bei ihrer Einführung wurden die Risiken kapitalgedeckter Alterssicherung systematisch verharmlost, die im individuellen Risiko der Fehlanlage und den kollektiven Risiken der - möglicherweise auch demografisch beeinflussten - Wirtschafts- und Renditeentwicklung sowie von Finanzmarkttur-

$\int 3 \mathrm{GSiG}$.

BSG, SozSich 2003, S. 207, 211; ebenso: Lenze, SF 2002, S. 33, 39; Neumann, DRV 1999, S. 393 , 404 f.; Bieback, KJ 1998, S. 162, 177.

Grütz, DRV 2004, S. 25, 33.

Vgl. $\int 154$ Abs. 3 Nr. 2 und Abs. 4 SGB VI in der Fassung des RV-Nachhaltigkeitsgesetzes.

Nürnberger/Stapf-Finé, SozSich 2004, S. 38, 41.

So auch Papier, S. 870: »verfassungswidrige Sondersteuer «.

Schmähl, in: VDR, S. 74, 77.

Vgl. Sackmann, GMH 1998, S. 713, 715.

So schon zur Rentenreform 1999 Sackmann, GMH 1998, S. 713, 720, der von einer »generationsspezifischen Deflationsspirale « der sozialen Sicherung spricht. 
bulenzen liegen. ${ }^{134}$ Die krisenhaften Entwicklungen von Pensionsfonds in Großbritannien, den USA, ${ }^{135}$ den Niederlanden und der Schweiz sowie bei den deutschen Lebensversicherungen haben inzwischen zur Ernüchterung beigetragen. ${ }^{136}$ Die Menschen für einen erheblichen Teil ihrer Altersvorsorgung unwägbaren Risiken und dem freien Spiel von ihnen nicht beeinflussbarer Kräfte auszusetzen, kann daher mit guten Gründen als Verstoß gegen das Sozialstaatsprinzip angesehen werden. ${ }^{137}$

Die Förderung ist zudem offensichtlich kein hinreichender Anreiz für ergänzende Alterssicherung. Vor allem begünstigt sie überdurchschnittlich diejenigen, die nicht vom Grundsicherungsniveau bedroht sind, nämlich die überdurchschnittlich verdienenden Rentenversicherten, und wird auch hauptsächlich von ihnen genutzt. ${ }^{138}$ Noch stärker als der Familienleistungsausgleich verfehlt die Riester-Förderung die eigentlich Bedürftigen. Sie bewirkt weniger eine gleichmäßige Lastenverteilung zwischen den Generationen ${ }^{139}$ als eher eine verstärkte Ungleichheit innerhalb der betroffenen Generation. Auch im internationalen Vergleich erscheinen die privaten Versicherungen und Fonds als eigentliche Profiteure steuerlicher Förderung der Altersvorsorge ${ }^{140}$ zumal Kunden und Staat bei ihnen einen weit höheren Anteil an Abschluss- und Verwaltungskosten mitfinanzieren müssen als bei öffentlichen Systemen. ${ }^{141}$ Die Verwaltungskosten der privaten Altersvorsorge liegen nach Untersuchungen der Weltbank im internationalen Vergleich zwischen $13,5 \%$ bis $26 \%$ der jährlichen Beiträge. ${ }^{142}$

Damit wird nicht negiert, dass ein demografisch bedingter höherer Rentnerquotient in dreißig Jahren zu höheren Rentenbeiträgen führen könnte. ${ }^{143}$ Es ist aber zu bestreiten, dass man heute schon vorhersagen kann, dass dann trotz eines prognostizierten Wirtschaftswachstums von durchschnittlich 1,7 \% ${ }^{144} \mathrm{im} \mathrm{Jahr}-$ also fast einer Verdoppelung des Sozialprodukts bis dahin ${ }^{145}$ - ein Beitrag von $22 \%$ die obere Belastungsgrenze darstellen würde. ${ }^{146}$ Es ist weiter zu bestreiten, dass

134 Vgl. Rieckhoff, DAngVers 2004, S. 118, 120: Wer 30 Jahre lang monatlich $50 €$ in einen deutschen Aktienfonds eingezahlt hat, konnte daraus im Jahre 2000 aus dem renditestärksten deutschen Aktienfonds eine Sofortrente von $1252 €$ (Männer) oder $1090 €$ (Frauen) erhalten. Bei gleicher Einzahlung in den renditeschwächsten Fonds betrug die Rente $2003151 €$ (Männer) und $132 €$ (Frauen). Der größte Teil des Unterschieds liegt im Verrentungszeitpunkt. Vgl. weiter Weeber, SF 2002, S. 28 ff.; Ruland, NZS 2002, S. 505, 508 f.; Unterhinninghofen, KJ 2002, S. 213, 218 f., 225 ff.; Fuchsloch, ZSR 2000, S. 700, 707 ff.; Scholz, DRV 2000, 578; Weeber, ZSR 1999, S. 506 ff.

Nach einer Studie der Unternehmensberatung Milliman USA haben die Pensionspläne der 100 größten US-amerikanischen Unternehmen eine Unterdeckung von 157 Milliarden Dollar, vgl. Oldag, SZ 3. April 2004.

136 Dazu bereits Ruland, NZS 2002, S. 505, 507. Nach einer jüngsten Untersuchung des Deutschen Instituts für Wirtschaftsforschung (DIW) sind trotz wachsender Unzufriedenheit der jüngeren Generation mit der Alterssicherung nur 8,5\% für ein überwiegend privatwirtschaftliches Alterssicherungssystem, $38 \%$ meinen, dass überwiegend der Staat verantwortlich sein solle und etwas mehr als $50 \%$ präferieren gemischte Verantwortlichkeit, Schwarze/Wagner/Wunder, DIW-Wochenbericht 22/2004, S. 4.

137 So Broß, JZ 2003, S. 874, 878.

138 Schwarze/Wagner/Wunder, DIW-Wochenbericht 22/2004, S. 6; Himmelreicher/Viebrok, WSI-Mitteilungen 2004, S. 11 ff.; Schmähl, DAngVers 2003, S. 349, 356 ff.

139 So die Regierungsfraktionen, BT-Drucks. 15/2149 vom 9. Dezember 2003, S. 17.

140 Sinfield, EJSS 2000, S. 137, 156.

141 Dies ist durch das Alterseinkünftegesetz noch verschärft worden, indem die Frist, über die Abschlussund Vertriebskosten bei geförderten Altersvorsorgeverträgen zu verteilen sind ( $\mathbb{1}$ Abs. 1 Satz 1 Nr. 8 Alt-ZertG), von zehn auf fünf Jahren verkürzt wurde, dazu Nürnberger/Perreng, SozSich 2004, S. $146,154$.

142 Robert Holzmann, Director Social Protection, Weltbank, DAngVers 2000, S. 410.

143 Dagegen ist daran zu erinnern, dass alle gegenwärtigen Probleme, den Rentenbeitrag stabil zu halten, arbeitsmarkt- und verteilungsbedingt sind, vgl. Deml, SozSich 20002, S. 411, 412. BT-Drucks. 15/2149 vom 9. Dezember 2003, S. 33.

145 Vgl. Bosbach, GMH 2004, 96, 101 f.; Nürnberger/Stapf-Finé, SozSich 2004, S. 38, 43; Ruland, DRV 2000, S. 733, 753.

146 So meint es der Gesetzgeber seit 2001 zu wissen, \$ 154 Abs. 3 Nr. 1 SGB VI; vgl. BT-Drucks. 15/2149, S. $33 \mathrm{ff}$. 
andere Finanzierungsmöglichkeiten bei steigendem Volkseinkommen unausgeschöpft bleiben müssten. Bei seriöser Betrachtung ist zudem der Rentner- oder Altenquotient nicht isoliert, sondern zusammen mit der Versorgungslast für Kinder und Jugendliche sowie Erwerbslose zu betrachten. ${ }^{147}$

\section{Gleichbeitssatz}

\section{a) Gleichbeit zwischen den Sicherungssystemen}

Die geschilderten Gerechtigkeitsprobleme für ein System, dessen Voraussetzungen allmählich verändert werden, stellen sich als Gleichheitsproblem, wenn sie in anderen Sicherungssystemen nicht auftreten. Ein Belastungs- und Leistungsvergleich zwischen Alterssicherungssystemen muss nicht auf die steuerrechtliche Behandlung beschränkt bleiben. ${ }^{148}$ Damit ist die Frage nach der Bürgerversicherung gestellt. ${ }^{149}$ Wenn die gesetzliche Rentenversicherung ein reales Problem hat, dann ist es die sinkende Lohnquote am Volkseinkommen. ${ }^{150} \mathrm{Ob}$ sich dieser Trend umkehrt, liegt im Wesentlichen außerhalb der Reichweite des Sozialgesetzgebers bei den Tarifparteien. Wohl aber wäre die adäquate Reaktion, den Sozialstaat an gesellschaftliche Veränderungen anzupassen, ohne das soziale Sicherungsziel aufzugeben. Hierzu könnte man weitere Personengruppen in die Rentenversicherung ${ }^{151}$ und zusätzliche Einkommen in die Beitragsbemessung einbeziehen. Seine Beamten ${ }^{152}$ und Abgeordneten ${ }^{153}$ in der Rentenversicherung zu sichern, wäre dem Staat erlaubt. Für noch nicht einbezogene Selbstständige besteht vielfach auch ein Sicherungsbedürfnis. ${ }^{154}$ Aber auch ein zusätzlicher Beitrag wäre zulässig, der auf Arbeitnehmerseite Vermögenseinkünfte, auf Arbeitgeberseite die Wertschöpfung belasten könnte. ${ }^{155}$ Er könnte auch die Gestalt einer Steuer haben, aus der ein zusätzlicher Zuschuss finanziert werden könnte. Die familienbezogenen Komponenten der Sozialversicherung sollten jedenfalls gesamtgesellschaftlich durch Steuern finanziert werden. ${ }^{156}$

Hein/Mülhaupt/Truger/Bartsch, WSI-Mitteilungen 2004, S. 291, 295 zeigen auf, dass der Anstieg der Belastungen bei Einbeziehung dieser Faktoren sehr viel geringer ausfällt. 2001 standen 100 Erwerbstätigen 96,5 Junge und Alte gegenüber, die zu versorgen waren. Würde 2010 Vollbeschäftigung erreicht, würde dieser wert erst 2025 wieder erreicht werden.

148 Vgl. dazu BVerfG vom 6. März 2002, BVerfGE 105, 73, 110 mit einem Belastungsvergleich der steuerlichen Behandlung von Altersrenten und Beamtenpensionen; zur Kritik an der steuerrechtsimmanenten Betrachtungsweise vgl. Nürnberger/Perreng, SozSich 2004, S. 156, 157; zur systemübergreifenden Gleichheit: Ruland, NVwZ 1995, S. 417, 426.

Im historischen Kontext: Stolleis, FS Zacher, S. 1081, 1096.

Vgl. ausführlich: Hein/Mühlhaupt/Truger/Bartsch, WSI-Mitteilungen 2004, S. 291, $296 \mathrm{ff}$.

151 Vgl. zur Verfassungsmäßigkeit der Sozialversicherung der selbstständigen Künstler und Publizisten BVerfG vom 8. April 1987, BVerfGE 75, 108; zur Verfassungsmäßigkeit der Pflichtversicherung der Landwirte: BVerfG vom 9. Februar 1977, BVerfGE 44, 70, 89. zur zulässigen Pauschalierung von Beiträgen in der Pflichtversicherung der Handwerker: BVerfG vom 11. Oktober 1972, BVerfGE 34, 62, 66; zur zulässigen Aufhebung der Pflichtversicherungsgrenze in der Rentenversicherung der Angestellten: BVerfG vom 14. Oktober 1970, BVerfGE 29, 221, 235 und BVerfGE 29, 245, 253; zur zulässigen Beitragsfinanzierung des Kindergeldes durch die Arbeitgeber BVerfG vom 10. Mai 1960, BVerfGE 11, 105, 117; zur Zulässigkeit der Pflichtmitgliedschaft von Ärzten im Versorgungswerk: BVerfG vom 25. Februar 1960, BVerfGE 10, 354, 361. Für die Ausweitung des Versichertenkreises: Nürnberger, SozSich 2003, S. 330, 336 f.; Lenze, NZS 2003, S. 505, 513; Engelen-Kefer/Franz/Schoch/ Wiesehügel, S. 42 ff. Für Zulässigkeit einer Volksversicherung: Ebsen, in: HS-RV, \$4, Rz. 47; aktuell: Bieback, SozSich 2003, S. 416 ff.; Jaeger, NZS 2003, S. 225, 232. 
Ein weiteres zentrales Gleichheitsproblem besteht zwischen den Geschlechtern. Immer noch erreichen Männer besser als Frauen das Sicherungsziel der Rentenversicherung. ${ }^{157}$ Würde die Alterssicherung stärker auf private Vorsorge gestützt, so würden Frauen auf Grund ihrer typischen Lebens- und Einkommenslage noch schlechter gestellt. ${ }^{158}$ Jede Beschränkung des Rentenanstiegs trifft darum Frauen stärker als Männer. ${ }^{159}$ Dazu kommt, dass in der privaten und zum Teil auch in der betrieblichen Altersvorsorge Frauen wegen ihrer höheren Lebenserwartung bisher einen höheren Beitrag zahlen müssen oder niedrigere Leistungen erhalten. Eine solche Differenzierung nach dem Geschlecht wäre in einem öffentlich-rechtlichen System unzulässig. ${ }^{160}$ Dass sie mit staatlicher Förderung betrieben werden und so bestehende Nachteile von Frauen fortschreiben und verschärfen darf, war ein gesetzgeberisches Versäumnis am Rande der Verfassungswidrigkeit und verstieß zudem bei betrieblicher Altersversorgung gegen das Gebot der Lohngleichheit. ${ }^{161}$ Der Gesetzgeber hat der wachsenden Kritik jetzt Rechnung getragen und mit dem Alterseinkünftegesetz eine vom Geschlecht unabhängige Kalkulation der geförderten Altersvorsorge von 2006 an vorgeschrieben. ${ }^{162}$ Jedenfalls verschränken sich in den unterschiedlichen Auswirkungen der Reformen auf männliche und weibliche Lebensverläufe die Probleme von Geschlechter- und Generationengerechtigkeit, ${ }^{163}$ und es wird einmal mehr deutlich, dass in einer Generation nur wenige Interessen homogen sind.

\section{c) Gleichbeit zwischen den Generationen?}

Nach allem bleibt außerhalb des Schutzes der Lebensgrundlagen wenig Raum für eine eigenständige Kategorie der Generationengerechtigkeit. Individuelle Gerechtigkeit nach den Kriterien Besitzstand, Bedarf und Leistung gegenüber den Mitgliedern aller Generationen erscheint ausreichend, um die Probleme der sozialen Sicherung rechtlich zu erfassen. ${ }^{164}$ Ein Ausgleich der Interessen zwischen Beitragszahlern und Rentnern lässt sich ohne weiteres im Rahmen von Inhalt und Schranken des Eigentums und allgemeiner Handlungsfreiheit vornehmen und bedarf nicht, wie Anne Lenze zutreffend feststellt, eines »aufgebauschten Gleichheitsgrundsatzes «. ${ }^{165}$ Nicht möglich und sinnvoll wäre, jede Veränderung auszuschließen, um Mitgliedern aller Generationen gleiche Sicherheit zu gleichen Bedingungen zu bieten. Die Schicksale, Lasten und Bedürfnisse der Generationen lassen sich nicht gegeneinander aufrechnen, weil sie jeweils besonders und einmalig

Vgl. bereits BVerfG vom 28. Januar 1987, BVerfGE 74, 163, 180 f.; Geissler, KJ 1997, S. 1 ff. Veil, WSI-Mitteilungen 2002, S. 91 ff.; Fuchsloch, ZSR 2000, S. 700, 710.

Nach BT-Drucks. 15/2149 vom 9. Dezember 2003, S. 38, wird das RV-Nachhaltigkeitsgesetz als "gleichstellungspolitisch ausgewogen « eingeschätzt.

Vgl. BVerfG vom 16. Juni 1981, BVerfGE 57, 335, 342. Art. $141 \mathrm{EGV}$; 612 Abs. 3 BGB; Hensche, ArbuR 2002, S. 167, 171.

Die Änderung ist erst im Finanzausschuss in Art. 5 des Alterseinkünftegesetzes durch eine entsprechende Änderung von $\ 1$ Abs. 1 Satz 1 Nr. 2 des Altersvorsorgeverträge-Zertifizierungsgesetzes aufgenommen worden, vgl. BT-Drucks. 15/2986 vom 28. April 2004, S. 56. Das Alterseinkünftegesetz ist nach einem Vermittlungsverfahren am 28. Mai 2004 im Bundestag gegen die Stimmen der CDU/ CSU-Fraktion angenommen, im Bundesrat am 11. Juni 2004 auch mit den Stimmen der Länder Sachsen und Hamburg beschlossen worden; vgl. Nürnberger/Perreng, SozSich 2004, S. 146, 154. Geissler, KJ 1997, S. 1, 13.

Dies löst den von Nürnberger, SozSich 2003, S. 330, 335 gesehenen Widerspruch auf; Von Lucke, Blätter für deutsche und internationale Politik 2003, S. 1055, 1059. 

nerationensolidarität setzt gerade voraus, dass sich die Generationen in ihrer Verschiedenheit in der Zeit anerkennen.

So kann von einem gesetzlichen Rentensystem so wenig wie von einer Volkswirtschaft oder einem Unternehmen erwartet werden, dass alle Rahmendaten in dreißig Jahren unverändert bleiben. Die Forderung nach gleich bleibenden Beiträgen $^{168}$ oder gleichen Renditen der Beiträge ${ }^{169}$ wird dem nicht gerecht und könnte nur in einer statischen Volkswirtschaft und letztlich auch nur in einer Diktatur verwirklicht werden. ${ }^{170} 20 \%$ Beitrag zu zahlen ist heute eine andere Belastung als es 1970 gewesen wäre und wird 2030 eine geringere Belastung sein als heute, weil Produktivität und Kaufkraft wachsen. ${ }^{171}$ Dies gilt umso mehr, als historische Besonderheiten bei der Einführung von Umlageverfahren oder nach Krisenzeiten einen Renditevergleich zwischen den Generationen systematisch verzerren und ein reifes, langfristig eingeführtes Umlageverfahren sich-ohne politische Eingriffe - stets an der Arbeitskräfte- und Produktivitätsentwicklung orientieren wird. ${ }^{172}$ Mit einer Gleichheit der Grundprinzipien und ihrer Ausformung zu jeweiligen Bedingungen wäre mehr gewonnen als mit einer Aufrechnung von Beiträgen und Leistungen über Jahrzehnte hinweg. ${ }^{173}$ In der Familie funktioniert es nicht anders: Wer seine pflegebedürftigen Eltern aufnimmt oder einen Heimplatz für sie sucht, rechnet nicht mit seinem früheren Taschengeld und den Wohnverhältnissen seiner Jugend auf, sondern versucht, Generationensolidarität nach den Möglichkeiten seiner Zeit auszugestalten.

Auch das Rentenalter kann verändert werden. Denn mit ihm wird im Grundsatz nur angezeigt, dass das versicherte Risiko typischer altersbedingter Einkommenslosigkeit eingetreten ist. Man kann so wenig verlangen, dass dies immer gleich bleiben soll, wie man von einer Unfallversicherung verlangen kann, dass es immer gleich viele Unfälle gibt. Eine langfristig angekündigte Anhebung des Rentenalters $^{174}$ als Reaktion auf steigende Lebenserwartung und bessere Gesundheit wäre eine systemadäquate Lösung. ${ }^{175}$ Es darf aber nicht verkannt werden, dass die Altersgrenze auch einen Bezug zur Ordnung des Arbeitsmarkts hat. ${ }^{176}$ Ihre Festsetzung darf nicht durch eine soziale Realität widerlegt werden, in der über 50jährige keine Arbeit mehr finden, gesundheitliche Belastungen am Arbeitsplatz nicht berücksichtigt werden und in der ein höheres Rentenalter nur eine faktische Rentenkürzung bedeuten würde. Längere Erwerbstätigkeit durch Diskriminierungs-

Nullmeier, in: VDR, S. 65, 70; Lenze, SF 2002, S. 33, 41; Ruland, DRV 2000, S. 733, 752; vgl. Sackmann, GMH 1998, S. 713, 716; LSG Nordrhein-Westfalen, NZS 2002, S. 265, 267: "Nach Auffassung des Senates handelt es sich bei den jetzigen Beitragszablern und den jetzigen Rentenempfängern nicht um wesentlich gleiche Personengruppen.«Vgl. aber die Definition der Generationengerechtigkeit bei Tremmel, in: SzRG, S. 34: »Generationengerechtigkeit ist erreicht, wenn die Chancen zukünftiger Generationen auf Befriedigung ibrer eigenen Bedürfnisse mindestens so gro $\beta$ sind wie die der heutigen Generation.«.

167 Ossenbühl, FS Zacher, S. 673, $682 \mathrm{ff}$.

168 So fordert es der Bundesrat, BT-Drucks. 15/2562 vom 25. Februar 2004, S. 9.

169 So gefordert von Tremmel, in: SzRG, S. 38, 43 und wohl auch Hebeler, DRV 2002, S. 270, 280; Zur Berechnung und zur Problematik von Renditevergleichen zwischen gesetzlicher und privater Alterssicherung: Schmähl, DAngVers 2003, S. 349, 359 ff.; Ruland, NZS 2002, S. 505, 509; Ohsmann/Stolz, DAngVers 2004, S. 56 ff.; vgl. Papier, S. 873.

Ruland, DRV 2000, S. 733, 751 f.; vgl. Nullmeier, KJ 1997, S. 261, 262 ff. zur »Altersgruppengerechtigkeit « und »Renditegerechtigkeit«: »unlösbar erscheinendes Anforderungsprofil.«. Vgl. Bosbach, GMH 2004, S. 96, 101 f.; Ruland, NZS 2002, S. 505, 510; Deml, SozSich 2002, S. 411, 417. Rabe/Langelüddeke, DAngVers 1999, S. 586, 587 unter Verweis auf neuere Untersuchungen der Weltbank. 
schutz im Arbeitsrecht, Weiterbildung und Rehabilitation im Sozialrecht ${ }^{177}$ und veränderte gesellschaftliche Mentalitäten zu erreichen, ${ }^{178}$ ist - neben besserer gesellschaftlicher Betreuung und Erziehung der Kinder und der dadurch ermöglichten Erwerbstätigkeit der Frauen - die wirklich zentrale Herausforderung des sozialen Rechtsstaats bei der Reform des Generationenverhältnisses.

\section{Ausblick}

Die Generationengerechtigkeit ist beim Schutz der natürlichen Lebensgrundlagen im Interesse noch nicht geborener Generationen zu Recht verfassungsrechtlich verankert und wartet dort noch auf ihre Konjunktur. Im Sozialstaat ist der Begriff - wie gezeigt - ebenso missverständlich wie missbrauchsanfällig. Ob er dort dennoch heimisch wird, hängt davon ab, ob der soziale Rechtsstaat den heute lebenden Generationen gerecht werden kann. Gleichheit über lange Zeiträume hinweg ist vom Grundgesetz nur für die Geltung seiner Prinzipien angeordnet worden: ${ }^{179}$ den Kern der Grundrechte, Föderalismus, Sozialstaat, Rechtsstaat, Demokratie. Entsprechend wird man im Recht der sozialen Sicherheit darauf achten müssen, dass über lange Zeit seine Prinzipien wie Leistungs- und Bedarfsgerechtigkeit erhalten bleiben, während sich deren rechtliche Ausformung ändern darf und muss.

\section{Literatur}

Adamy, Wilhelm: Herausforderungen einer älter werdenden Erwerbsbevölkerung, in: Engelen-Kefer, Ursula/Wiesehügel, Klaus (Hrsg.), Sozialstaat - solidarisch, effizient, zukunftssicher, Hamburg 2003, S. 86-103.

Bäcker, Gerhard: Armut und Unterversorgung im Kindes- und Jugendalter: Defizite der sozialen Sicherung, in: Butterwegge, Christoph (Hrsg.), Kinderarmut in Deutschland, Frankfurt am Main, 2. A., 2000, S. 244-269.

Beaucamp, Guy: Das »Ozongesetz« vor den Verwaltungsgerichten, JA 1999, S. 170-175.

Becker, Ulrich: Generationengerechtigkeit als juristisches Problem, in: VDR (Hrsg.), Generationengerechtigkeit - Inhalt, Bedeutung und Konsequenzen für die Alterssicherung, DRV-Schriften 51, Bad Homburg 2004, S. 56-64.

Behrends, Andrea: Schutz der Umwelt (Art. 20 a GG) und gesetzgeberische Behandlung der regenerativen Energieerzeugung, KJ 2000, S. 376-394.

Bieback, Karl-Jürgen: Verfassungsrechtliche Aspekte einer Bürgerversicherung: Der Bund hat die Kompetenz zur Einführung einer umfassenden Versicherung, Soziale Sicherheit 2003, S. 416-425.

Bieback, Karl-Jürgen: Sicherheit im Sozialstaat, Kritische Justiz (KJ) 1998, S. 162-178.

Birg, Herwig: Dynamik der demographischen Alterung, Bevölkerungsschrumpfung und Zuwanderung in Deutschland, Aus Politik und Zeitgeschichte Nr. 20/2003, S. 6-16.

Bogs, Harald: Art. 14 GG (Eigentum) als Vertrauensschutz-Basisnorm für Rentenversicherte?, in: Ruland, Franz/Maydell, Bernd Baron von/Papier, Hans-Jürgen (Hrsg.), Verfassung, Theorie und Praxis des Sozialstaats. Festschrift für Hans F. Zacher zum 70. Geburtstag, Heidelberg 1998, S. 65-83.

Bosbach, Gerd: Demografische Entwicklung - nicht dramatisieren, Gewerkschaftliche Monatshefte (GMH) 2004, S. 96-103.

Blüggel, Jens: Verstößt die geplante Nichtanrechnung gegen das Eigentumsgrundrecht?, Soziale Sicherheit (SozSich) 2004, S. 61-68.

Blüm, Norbert: Generationenvertrag, in: Deufel, Konrad/Wolf, Manfred (Hrsg.), Ende der Solidarität?, Freiburg 2003, S. 104-112.

Bogs, Walter/Achinger, Hans/Meinhold, Helmut/Neundörfer, Ludwig/Schreiber, Wilfried: Sozialenquete: Soziale Sicherung in der Bundesrepublik Deutschland, Stuttgart 1967. 
Broß, Siegfried: Daseinsvorsorge - Wettbewerb - Gemeinschaftsrecht, Juristenzeitung (JZ) 2003, S. 874-879.

Bull, Hans Peter: Umsteuern im Beamtenrecht - aber wie?, Die öffentliche Verwaltung (DÖV) 1995, S. 592-596.

Däubler-Gmelin, Herta: Die Verankerung von Generationengerechtigkeit im Grundgesetz - Vorschlag für einen erneuerten Art. 20 a GG, Zeitschrift für Rechtspolitik (ZRP) 2000, S. 27-28.

Deml, Jörg: Gerecht und sicher, SozSich 2002, S. 411-418.

Ebert, Thomas: Beutet der Sozialstaat Familien aus?, in: Butterwegge, Christoph/Klundt, Michael (Hrsg.), Kinderarmut und Generationengerechtigkeit, Opladen 2002, S. 99114.

Ebsen, Ingwer: Verfassungs- und europarechtliche Vorgaben $(\$ 4)$, in: Schulin, Bertram (Hrsg.), Handbuch des Sozialversicherungsrechts, Bd. 3, Rentenversicherungsrecht (HS-RV), München 1999, S. 133-163.

Eggen, Bernd: Demographische Depressionen, SF 2002, S. 255-259.

Eichenhofer, Eberhard: Beitragsvergünstigungen für Eltern in der Rentenversicherung?, Mitteilungen der Landesversicherungsanstalt Oberfranken und Mittelfranken 2001, S. 719-727.

Engelen-Kefer, Ursula/Franz, Nadine/Schoch, Manfred/Wiesehügel, Klaus: Minderheitengutachten zu den Vorschlägen der Rürup-Kommission, in: Engelen-Kefer, Ursula/ Wiesehügel, Klaus (Hrsg), Sozialstaat - solidarisch, effizient, zukunftssicher, Hamburg 2003.

Esping-Andersen, Gösta: Aus reichen Kindern werden reiche Eltern - Vorschläge, wie die Politik dem Phänomen der sozialen Vererbung entgegensteuern kann, Frankfurter Rundschau vom 20. 12. 2003.

Fuchsloch, Christine: Moderne Anforderungen an eine Alterssicherung und das Rentenreformmodell der Bundesregierung, Zeitschrift für Sozialreform (ZSR) 2000, S. 700717.

Geissler, Birgit: Netz oder Sieb? Generationenkonflikt und Geschlechterkonflikt in der aktuellen Krise des Sozialstaats, Kritische Justiz (KJ) 1997, S. 1-14.

Göckenjan, Gerd: Das Alter würdigen - Altersbilder und Bedeutungswandel des Alters, Frankfurt am Main 2000.

Grütz, Jens: Die Reformmaßnahmen zur Stabilisierung des Beitragssatzes zur Rentenversicherung, DRV 2004, S. 25-37.

Haass, Jörg: Neue Systemgerechtigkeit? Zum Gleichheitsverstoß der Pflegeversicherung, KJ 2002, S. 104-110.

Häberle, Peter: Das Verfassungsrecht für künftige Generationen - Die »andere « Form des Gesellschaftsvertrages: der Generationenvertrag, in: Ruland, Franz/Maydell, Bernd Baron von/Papier, Hans-Jürgen (Hrsg.), Verfassung, Theorie und Praxis des Sozialstaats. Festschrift für Hans F. Zacher zum 70. Geburtstag, Heidelberg 1998, S. 215-234.

Hebeler, Timo: Der Gleichheitssatz des Art. 3 I GG als Schlüssel für die Pflicht einer generationengerechten Ausgestaltung des Rechts der gesetzlichen Altersrenten, DRV 2002, S. 270-281.

Hein, Eckhard/Mülhaupt, Bernd/Truger, Achim/Bartsch, Klaus: Demographische Entwicklung - Ein Standortproblem?, WSI-Mitteilungen 2004, S. 291-305.

Heller, Hermann: Staatslehre, Leiden, 1934.

Hensche, Detlef: Gleiche Arbeit - ungleiche Rente?, Arbeit und Recht (ArbuR) 2002, S. $167-171$.

Himmelreicher, Ralf K./Viebrok, Holger: Staatliche Förderung der privaten Altersvorsorge - einige verteilungspolitische Aspekte, WSI-Mitteilungen 2004, S. 11-18.

Igl, Gerhard: Die Risiken im Alter, in: Cramer, Jörg/Förster, Wolfgang/Ruland, Franz (Hrsg.), Handbuch zur Altersversorgung, Frankfurt am Main 1998, S. 2741.

Jaeger, Renate: Die Reformen in der gesetzlichen Sozialversicherung im Spiegel der Rechtsprechung des Bundesverfassungsgerichts, Neue Zeitschrift für Sozialrecht (NZS) 2003, S. 225-234.

Kähler, Christoph: Generationengerechtigkeit-Biblisch-theologische Anmerkungen, in: VDR (Hrsg.), Generationengerechtigkeit - Inhalt, Bedeutung und Konsequenzen für die Alterssicherung, DRV-Schriften 51, Bad Homburg 2004, S. 32-38.

Kaufmann, Franz Xaver: Varianten des Wohlfahrtsstaats, Frankfurt am Main 2003.

Kingreen, Thorsten: Das Sozialstaatsprinzip im europäischen Verfassungsverbund, Tübingen 2003.

Kirchhof, Paul: Die Aufgaben des Bundesverfassungsgerichts in Zeiten des Umbruchs, NJW 1996, S. 1497-1511. 
Kistler, Ernst: Demographischer Wandel und Arbeitsmarkt - Die Debatte muss ehrlicher werden, WSI-Mitteilungen 2004, S. 71-77.

Köhler-Rama, Tim: Kinderzahlabhängige Beiträge in der gesetzlichen Rentenversicherung: Rückschritt statt Fortschritt, Die Angestelltenversicherung (DAngVers) 2002, S. 449-454.

Kotulla, Michael: Verfassungsrechtliche Aspekte im Zusammenhang mit der Einführung eines Umweltgrundrechts im Grundgesetz, KJ 2000, S. 22-34.

Lauterbach, Wolfgang/Lange, Andreas/Becker, Rolf: Armut und Bildungschancen, in: Butterwegge, Christoph/Klundt, Michael (Hrsg.), Kinderarmut und Generationengerechtigkeit, Opladen 2002, S. 153-172.

Leinert, Johannes: Freiwillige Altersvorsorge: Finanzielle Förderung ist nicht alles, SF 2004, S. 55-62.

Lenze, Anne: Teilhabeberechtigung statt Renteneigentum: Überlegungen zu einem neuen verfassungsrechtlichen Konzept der gesellschaftlichen Alterssicherung, Sozialer Fortschritt (SF) 2002, S. 33-42.

Lenze, Anne: Rentenreform zwischen Eigentumsschutz und Gleichheitssatz, Neue Zeitschrift für Sozialrecht (NZS) 2003, S. 505-513.

Lucke, Albrecht von: Generationengerechtigkeit als Kampfbegriff, Blätter für deutsche und internationale Politik 2003, S. 1055-1059.

Lumer, Christoph: Prinzipien der Generationengerechtigkeit, in: Stiftung für die Rechte zukünftiger Generationen (SzRG), Handbuch Generationengerechtigkeit, München, 2. A. 2003, S. 105-124.

Lux-Wesener, Christina: Generationengerechtigkeit im Grundgesetz?, in: Stiftung für die Rechte zukünftiger Generationen (SzRG), Handbuch Generationengerechtigkeit, München, 2. A. 2003, S. 405-440.

Mackenroth, Gerhard: Die Reform der Sozialpolitik durch einen deutschen Sozialplan (1952), Auszug bei Ganßmann, Heiner: Politische Ökonomie des Sozialstaats, Münster 2000, S. 23.

Murswiek, Dietrich: Staatsziel Umweltschutz (Art. 20 a GG) - Bedeutung für Rechtsetzung und Rechtsanwendung, NVwZ 1996, S. 222-230.

Neumann, Volker: Konsolidierungsmaßnahmen im Rentenversicherungsrecht contra Grundrechtsschutz, Deutsche Rentenversicherung (DRV) 1999, S. 393-405.

Nürnberger, Ingo: Kurzfristige Reparaturen, längerfristige Einschränkungen - Die geplanten Änderungen bei der Rente, Soziale Sicherheit (SozSich) 2003, S. 330-337.

Nürnberger, Ingo/Stapf-Finé, Heinz: Drastische Einschnitte stellen Versicherungssystem in Frage, SozSich 2004, S. 38-43.

Nürnberger, Ingo/Perreng, Martina: Die künftige Besteuerung von Alterseinkünften Die neuen Regelungen zur privaten und betrieblichen Vorsorge, SozSich 2004, S. 146157.

Nullmeier, Frank: Über eine neuerliche Rentenreform, KJ 1997, S. 261-276.

Nullmeier, Frank: Generationengerechtigkeit - aus politikwissenschaftlicher Sicht, in: VDR (Hrsg.), Generationengerechtigkeit - Inhalt, Bedeutung und Konsequenzen für die Alterssicherung, DRV-Schriften 51, Bad Homburg 2004, S. 65-73.

Ohsmann, Sabine/Stolz, Ulrich: Entwicklung der Rendite in der gesetzlichen Rentenversicherung, DAngVers 2004, S. 56-62.

Oldag, Andreas: Betriebsrenten überfordern US-Konzerne - Angst vor dem Abstieg, Süddeutsche Zeitung vom 3. April 2004.

Ossenbühl, Fritz: Soziale Gleichheit in der Zeit, in: Ruland, Franz/Maydell, Bernd Baron von/Papier, Hans-Jürgen (Hrsg.), Verfassung, Theorie und Praxis des Sozialstaats. Festschrift für Hans F. Zacher zum 70. Geburtstag, Heidelberg 1998, S. 673-688.

Papier, Hans-Jürgen: Verfassungsrechtliche Vorgaben für die Alterssicherung, in: Cramer, Jörg/Förster, Wolfgang/Ruland, Franz (Hrsg.), Handbuch zur Altersversorgung, Frankfurt am Main 1998, S. 855-890.

Peters, Heinz-Joachim: Art. 20 a GG - Die neue Staatszielbestimmung des Grundgesetzes, NVwZ 1995, S. 555-557.

Rabe, Birgitta/Langelüddeke, Anne: Gewichtung der Kapitaldeckung im Drei-SäulenKonzept der Alterssicherung - Kehrtwende der Weltbank?, DAngVers 1999, S. 586588.

Radbruch, Gustav: Rechtsphilosophie, Heidelberg 1999.

Reuter, Norbert: Generationengerechtigkeit als Richtschnur der Wirtschaftspolitik?, in: Butterwegge, Christoph/Klundt, Michael (Hrsg.), Kinderarmut und Generationengerechtigkeit, Opladen 2002, S. 81-98. 
Rieckhoff, Christian: Individuelle Planbarkeit der privaten Altersvorsorge, DAngVers 2004, S. 118-127.

Römer, Robin: Rentenversicherung: Beschränkte Aussagefähigkeit von Prognosen, SozSich 2003, S. 188-190.

Ruland, Franz: Familie und Alterssicherung, FamRZ 2004, S. 493-500.

Ruland, Franz: Die Rentenreform unter besonderer Berücksichtigung der staatlich geförderten zusätzlichen Altersvorsorge, NZS 2002, S. 505-510.

Ruland, Franz: Das BVerfG und der Familienlastenausgleich in der Pflegeversicherung, NJW 2001, S. 1673-1678.

Ruland, Franz: Solidarität und Individualität, DRV 2000, S. 733-757.

Ruland, Franz: Zur Zukunft von gesetzlicher Rentenversicherung und Beamtenversorgung, NVwZ 1995, S. 417-426.

Sackmann, Reinhold: Generationenverhältnis und Gerechtigkeit - das Beispiel Rentenversicherung, GMH 1998, S. 713-720.

Sacksofsky, Ute: Steuerung der Familie durch Steuern, NJW 2000, S. 1896-1903.

Scheiwe, Kirsten: Kinderkosten und Sorgearbeit im Recht, Frankfurt am Main 1999.

Schlenker, Rolf-Ulrich: Soziales Rückschrittsverbot und Grundgesetz, Berlin (West) 1986.

Schmähl, Winfried: Wem nutzt die Rentenreform?, DAngVers 2003, S. 349-363.

Schmähl, Winfried: Plädoyer für eine einheitliche und verständliche Rentenformel, SF 2001, S. 2-6.

Scholz, Wolfgang: Alterssicherung und Kapitaldeckung, DRV 2000, S. 578-596.

Schulin, Bertram/Igl, Gerhard: Sozialrecht, 7. A., Düsseldorf 2001.

Schwarze, Johannes/Wagner, Gert/Wunder, Christoph: Alterssicherung: Gesunkene Zufriedenheit und Skepsis gegenüber privater Vorsorge, DIW-Wochenbericht 22/2004.

Sellmann, Christian: Die eigentumsrechtliche Inhalts- und Schrankenbestimmung - Entwicklungstendenzen, NVwZ 2003, S. 1417-1423.

Sinfield, Adrian: Tax Benefits in Non-State Pension, European Journal of Social Security 2000, S. 137-167.

Steinberg, Rudolf: Verfassungsrechtlicher Umweltschutz durch Grundrechte und Staatszielbestimmung, NJW 1996, S. 1985-1994.

Stolleis, Michael: Industrielle Revolution und Sozialversicherung, in: Ruland, Franz/ Maydell, Bernd Baron von/Papier, Hans-Jürgen (Hrsg.), Verfassung, Theorie und Praxis des Sozialstaats. Festschrift für Hans F. Zacher zum 70. Geburtstag, Heidelberg 1998, S. 1081-1100.

Tennstedt, Florian: Generationengerechtigkeit in Deutschland - Anmerkungen aus historischer Perspektive, in: VDR (Hrsg.), Generationengerechtigkeit - Inhalt, Bedeutung und Konsequenzen für die Alterssicherung, DRV-Schriften 51, Bad Homburg 2004, S. 51-55.

Tremmel, Jörg, Institutionelle Verankerung der Rechte nachfolgender Generationen, ZRP 2004, S. 44-46.

Tremmel, Jörg: Generationengerechtigkeit - Versuch einer Definition, in: Stiftung für die Rechte zukünftiger Generationen (SzRG), Handbuch Generationengerechtigkeit, München, 2. A. 2003, S. 27-80.

Tremmel, Jörg: Positivrechtliche Verankerung der Rechte zukünftiger Generationen, in: Stiftung für die Rechte zukünftiger Generationen (SzRG), Handbuch Generationengerechtigkeit, München, 2. A. 2003, S. 349-382.

Ulmer, Matthias: Im Schatten der Scheinselbstständigkeit, ZRP 2001, S. 257-260.

Unterhinninghofen, Hermann: Rotgrünes Rentenprojekt, KJ 2002, S. 213-227.

Veil, Mechthild: Die Riester-Rente: Geschlechterspezifische Wirkungen der privaten und betrieblichen Vorsorge, WSI-Mitteilungen 2002, S. 91-97.

Wander, Hilde: Der Geburtenrückgang in Westeuropa wirtschaftlich gesehen, Kiel 1971.

Weeber, Joachim: Die Bedeutung der Finanzmärkte für die Stabilität der Alterssicherung, SF 2002, S. 28-33.

Weeber, Joachim: Auswirkungen von Finanzmarktturbulenzen auf den Sozialstaat, ZSR 1999, S. 506-525.

Welti, Felix: Abgeordnete in die Sozialversicherung?, Zeitschrift für Parlamentsfragen (ZParl) 2000, S. 254-274.

Welti, Felix: Bedürfnis nach sozialem Schutz wächst - Sozialversicherung für Selbstständige öffnen, Soziale Sicherheit 2001, S. 223-229.

Zacher, Hans F.: Abhandlungen zum Sozialrecht, Heidelberg 1993. 